Cahiers d'études africaines

\title{
Les métamorphoses des « relations à plaisanteries 》
}

Un nouvel enjeu politique dans la construction des États-nations *

\section{Marie-Aude Fouéré}

\section{CpenEdition}

\section{Journals}

Édition électronique

URL : http://journals.openedition.org/etudesafricaines/5430

DOI : 10.4000/etudesafricaines.5430

ISSN : $1777-5353$

Éditeur

Éditions de l'EHESS

Édition imprimée

Date de publication : 25 juin 2005

Pagination : 389-430

ISBN : 978-2-7132-2048-7

ISSN : 0008-0055

Référence électronique

Marie-Aude Fouéré, "Les métamorphoses des « relations à plaisanteries » », Cahiers d'études africaines [En ligne], 178 | 2005, mis en ligne le 30 juin 2008, consulté le 10 décembre 2020. URL : http://

journals.openedition.org/etudesafricaines/5430; DOI : https://doi.org/10.4000/etudesafricaines.5430

Ce document a été généré automatiquement le 10 décembre 2020.

(C) Cahiers d'Études africaines 


\title{
Les métamorphoses des « relations à plaisanteries »
}

\author{
Un nouvel enjeu politique dans la construction des États-nations *
}

\section{Marie-Aude Fouéré}

1 L'expression générique de « relations à plaisanteries » est apparue en ethnologie au début $\mathrm{du} \mathrm{xx}^{\mathrm{e}}$ siècle. Elle a été utilisée pour désigner des pratiques sociales variées, géographiquement éloignées, que le comparatisme fonctionnaliste des années 1940 s'est empressé d'unifier au vu de leur similarité structurelle. Longtemps cantonnées dans le champ disciplinaire de l'ethnologie, où elles se sont imposées comme un classique des monographies à partir des années 1960, ces pratiques sont en passe de devenir un des grands thèmes politiques rassembleurs dans l'Afrique de ce début de siècle. À la recherche d'un fondement proprement africain du lien social, nombre d'intellectuels et d'hommes politiques voient en effet dans les relations à plaisanteries un savoir endogène à promouvoir dans la prévention des conflits interethniques.

2 L'objectif de cet article est de montrer comment les ethnologues du siècle dernier aussi bien que les élites africaines actuelles ont été amenés à ignorer la dimension agonisitique qui fonde les pratiques désignées par l'expression de relations à plaisanteries. Pour cela, il convient, dans une optique déconstructiviste, de revenir sur la manière dont un ensemble de pratiques a été construit comme une « coutume » ou une «tradition». Par ces termes, on renvoie au paradigme ethnologique classique qui conçoit les manières de faire exotiques comme un ensemble homogène de pratiques pétrifiées, transmises telles quelles d'une génération à l'autre et utiles à la reproduction à l'identique de l'organisation sociale. L'adoption d'un point de vue historique et politique permet dans un second temps de compléter cette analyse critique de la "raison ethnologique». Dévoilant les logiques historiques de production et de reproduction des relations à plaisanteries comme pratiques de pouvoir, cette deuxième partie met en lumière le poids des rapports de force entre les organisations sociopolitiques précoloniales et les enjeux identitaires qui les sous-tendent. L'analyse des modalités concrètes de mise en œuvre de ces pratiques dans un contexte africain moderne vient compléter cette réflexion générale sur la question du pouvoir au cœur des pratiques sociales. Dans ce cadre, les situations 
d'interaction constituent un lieu d'observation particulièrement pertinent des logiques d'émergence de comportements et de paroles qualifiés de relations à plaisanteries par les locuteurs. Enfin, l'étude du processus actuel de réappropriation du concept de relations à plaisanteries par les élites africaines permet de mettre à jour son ancrage dans les discours ethnologiques passés et sa visée hautement politique. Si c'est avant tout le cas de la Tanzanie qui est ici discuté, un va-et-vient constant entre Afrique de l'Est et Afrique de l'Ouest va permettre de dresser un panorama général des pratiques et des discours relatifs à cet impensé du sens commun ethnologique qu'est la catégorie de relations à plaisanteries.

Que sont les « relations à plaisanteries »?

3 Utani en Tanzanie, senankuya ${ }^{1}$ au Mali, dendiragu et dakure au Burkina Faso, banungwe en Zambie : ces termes constituent quelques exemples parmi la multitude de désignations locales, parfois strictement régionales, d'autres fois transnationales, qui ont été regroupées sous le concept de « relations à plaisanteries » (ou «joking relationships » en anglais ${ }^{2}$ ). Ils apparaissent pour la première fois dans les écrits des Européens datant de la fin du $\mathrm{xIX}^{\mathrm{e}}$ siècle. Ainsi, concernant le Tanganyika, l'administrateur et explorateur allemand Peter Reichar mentionne le terme swahili utani dans un article du Deutsche Kolonialzeitung en 1890. Selon l'auteur, ce terme désigne une coutume "bien étrange » (" eigentümliche Sitte ») qui lie des " tribus » ("Stämme») du nord-est du Tanganyika, mais aussi des populations du centre de ce territoire. Au début du $\mathrm{xx}^{\mathrm{e}}$ siècle, seuls quelques articles traitant de l'utani sont publiés (Dale 1896 ; Abdy 1924). C'est à partir des années 1930-1940 que ce phénomène attire l'attention d'un nombre croissant d'observateurs du Tanganyika et qu'il commence à être traduit par l'expression " relations à plaisanteries ». En 1935, le révérend McVicar publie dans la revue Primitive Man un article sur une population de l'est du Tanganyika, nommée les Waluguru, qui traite de l'utani (localement nommé ugongo) entre clans. En 1937, le révérend T. V. Scrivenor fait paraître un rapport sur les relations à plaisanteries entre clans au sein des populations du sud-est du territoire. Enfin, ce sont les parutions des articles de l'administrateur britannique F. Pedler (1940) dans la revue Africa et du botaniste anglais R. E. Moreau (1941, 1944), reconverti à l'ethnologie, dans la revue Tanganyika Notes and Records et dans Africa, qui entérinent l'intérêt croissant des observateurs étrangers pour les relations à plaisanteries au Tanganyika. Les travaux ethnographiques menés parallèlement dans le reste de l'Afrique (Soudan français : Delafosse 1912 ; Humblot 1918 ; Labouret 1929; Doumbia 1936. Rhodésie du Nord: Junod 1962; Dale \& Smith 1920; Melland 1967; Richards 1937; Doke 1931 ; White 1955 ; Stefaniszyn 1964. Nigeria : Meek 1925 ; Haffenden 1930) confirment que les phénomènes de relations à plaisanteries deviennent des objets de recherche privilégiés de l'ethnologie.

4 Avant d'opérer un travail critique sur les modes de construction de pratiques exotiques variées en « coutume», il convient de présenter les informations qui ont été accumulées au cours du temps par les premiers observateurs, qu'ils soient explorateurs, administrateurs ou bien ethnologues. Pour cela, je résume ces informations avec l'objectif de restituer la "matrice informative ", c'est-à-dire un tableau cohérent des données recueillies, qui a été sous-jacente à la majorité des élaborations théoriques successives.

5 Selon une définition classique, l'expression de relations à plaisanteries renvoie à des relations spécifiques qui s'instaurent soit entre certains membres d'un groupe de parenté ${ }^{3}$, soit entre clans ou lignages, soit entre ethnies ${ }^{4}$. Ces relations impliquent l'adoption de comportements particuliers tels l'énonciation de plaisanteries ou de moqueries et 
l'exécution d'actes d'entraide. Ainsi, les textes ethnologiques portant sur la parenté à plaisanteries en Tanzanie indiquent qu'un petit-fils peut appeler sa grand-mère «ma femme » et une petite-fille son grand-père «mon mari ». En retour, un grand-père appelle sa petite-fille «ma femme » et une grand-mère son petit-fils «mon mari ». Les beaux-frères et belles-sœurs, aussi bien que les cousins croisés, peuvent se faire des avances sexuelles sous forme de plaisanteries ou recourir à un langage obscène. Enfin, un homme peut plaisanter avec son oncle maternel, se moquer de lui et lui voler des affaires. Dans le second type de relations à plaisanteries distingué par l'ethnologie classique, les membres de lignages ou de clans liés se doivent entraide ou assistance dans les moments difficiles. Les partenaires à plaisanteries conduisent les funérailles d'un membre du groupe avec lequel ils sont liés. Ils participent aux dépenses, creusent la tombe et enterrent le mort; ils préparent la cuisine et nettoient la maison de la famille endeuillée. Certains auteurs précisent que les partenaires à plaisanteries sont présents lors de la cérémonie d'investiture des chefs de clan avec lesquels ils ont établi des relations à plaisanteries. Ils effectuent en outre les rites funéraires de ces chefs. La caractéristique la plus marquante de ces relations renvoie aux comportements des partenaires à plaisanteries. Quand ces derniers se rencontrent, ils s'insultent ou se raillent, et peuvent aller jusqu'à l'injure ou aux coups. Tout individu peut entrer chez son partenaire à plaisanteries, boire les réserves de bière et se servir dans les stocks de nourriture. Il peut réclamer des habits portés à l'envers ou des vêtements qui sèchent sur le toit d'une hutte, ainsi que des vêtements neufs portés sans avoir été lavés. Au cours des funérailles, il arrive que les partenaires à plaisanteries s'emparent d'un poulet, se couchent dans une tombe et refusent d'en sortir. Ils demandent généralement une petite pièce pour cesser leurs abus. Enfin, les mêmes comportements ont été répertoriés par les premiers observateurs dans le cadre des relations à plaisanteries entre groupes ethniques. Outre la participation aux funérailles, le vol rituel d'objets et la possibilité de tenir des propos moqueurs ou injurieux, les devoirs majeurs évoqués consistent à offrir l'hospitalité à tout voyageur membre d'une ethnie à plaisanteries ainsi que lui proposer repas et aide financière. Indiquons pour finir que les pratiques de relations à plaisanteries décrites dans d'autres régions d'Afrique partagent des caractéristiques très similaires à l'utani du Tanganyika.

6 Maintenant qu'ont été présentés quelques aspects de ce qu'il est convenu d'appeler les « relations à plaisanteries ", il faut expliquer comment l'ethnologie classique a dépouillé ces pratiques de la réalité des rapports de pouvoir qui les fondent, insistant au contraire sur le respect des règles sociales rigides censées assurer le bon fonctionnement de la société. Les réflexions qui vont être développées traiteront plus spécifiquement des relations entre entités sociopolitiques (ce que l'ethnologie classique nomme couramment « clans » ou « ethnies »).

La construction savante d'une « coutume africaine intertribale »

7 Au fil des lectures portant sur le thème des relations à plaisanteries, on ne peut manquer de remarquer un consensus troublant entre les auteurs du début du $x^{e}$ siècle jusqu'à la fin des années 1970, parfois même jusqu'à aujourd'hui. Ce consensus porte sur les modes d'analyses et les interprétations qui ont été faites de ces pratiques.

La prégnance du modèle juridique

8 En premier lieu, on constate que les descriptions classiques des relations à plaisanteries sont caractérisées par une rhétorique juridique, que ce soit à propos de l'utani en Afrique de l'Est ou des pratiques similaires en Afrique occidentale. Ainsi, les informations que 
l'administrateur colonial Maurice Delafosse fournit en 1912 sur la senankuya au Soudan français sont présentées sous la forme de la règle: les sénankoun, c'est-à-dire les partenaires à plaisanteries, ont le «droit de s'injurier», «ils doivent se porter assistance ». Dans un article paru en 1918, P. Humblot rend compte de l'alliance senankuya entre groupes ethniques en déployant une terminologie juridique encore plus prononcée que chez Delafosse. Par exemple, l'auteur évoque des clans sanan'koun qui sont «moralement astreints, toutes les fois que l'occasion se présente, d'échanger des cadeaux, de se prêter mutuellement secours dans la misère, assistance dans l'adversité " (Humblot 1918 : 532). La même rhétorique juridique se perpétue à partir des années 1930 dans les travaux des premiers ethnologues de terrain. Ainsi, dans son article de 1929, Henri Labouret s'inscrit dans ce courant interprétatif fondé sur la codification juridique des pratiques. Dans un ouvrage ultérieur, il explique ainsi que la sanaku ya [sic] « consiste en l'obligation de se plaisanter et de s'insulter [...]. Les coups sont admis dans des circonstances fixées par la coutume» (Labouret 1934: 101). Denise Paulme (1939) et Germaine Dieterlen (1951) reprennent également cette perspective sans la remettre en cause. La prédominance du juridique transparait aussi dans les écrits portant sur l'Afrique de l'Est, mais de manière moins affirmée qu'en Afrique de l'Ouest. En effet, à partir des années 1920, la présentation de l'utani en termes de droits et de devoirs vient remplacer les témoignages des premiers observateurs qui se présentaient comme des descriptions objectives rédigées au présent ethnologique. Les articles de F. Pedler (1940): « It would be interesting to know what assistance, if any, is due from one mtani ${ }^{5}$ to another », de R. E. Moreau (1944) : « Mutual obligations » of utani, « the rule » of utani, ou de J. B. Christensen (1963) : "It must be viewed in terms of duties and obligations", témoignent de ce nouveau tournant en anthropologie. Au final, c'est bien une perspective juridique qui a gagné l'ensemble des travaux concernant les pratiques dites de relations à plaisanteries.

De cette perspective juridique découle une vision statique du fonctionnement des groupes sociaux. Dans cette logique, puisque tout est codifié et que les individus agissent selon les lois du groupe sans jamais les contourner ni les violer, il s'ensuit que le travail de l'ethnologue est la détermination de ces lois et des modalités de leur application. Pourtant, ce qu'ignore cette perspective normative, c'est le hiatus entre théorie et pratique. Recourir au vocabulaire du droit est une facilité stylistique et stratégique qui évite d'avoir à étudier non pas ce que les individus disent faire, mais ce qu'ils font (Bourdieu 1972). En outre, une telle approche laisse de côté la dimension conflictuelle qui est au cœur des pratiques sociales. Elle propose une vision normative de la vie en groupe qui évacue les tensions intergénérationnelles, les conflits de genre, les oppositions entre groupes sociaux au profit d'un tout fonctionnel qui repose sur une obéissance aveugle à un pouvoir régulateur tout-puissant. Si la perspective juridique a été décisive dans l'avènement du paradigme structuralo-fonctionnaliste en ethnologie, elle a aussi alimenté les théories symbolistes (Amselle 1996a). Concernant notre objet d'étude, Alfred RadcliffeBrown et Marcel Griaule sont les tenants respectifs de ces deux approches, dont les points communs sont frappants malgré des orientations théoriques opposées.

Les principaux modèles d'analyse

Dans deux articles successivement parus dans la revue Africa en 1940 et $1949{ }^{6}$, RadcliffeBrown fonde son analyse des relations à plaisanteries au sein du groupe familial sur la terminologie de parenté. Selon l'auteur, les relations à plaisanteries sont l'exact opposé des relations dites «d'évitement » entre membres du groupe familial, c'est-à-dire des 
relations de respect fort qui induisent des interdits de rencontres ou d'échanges de paroles. Elles permettraient d'évacuer les contraintes psychologiques qu'impose un ordre social rigide, et donc de maintenir le bon fonctionnement du groupe social. Elles constituent une "soupape de sécurité " pour chaque être humain et pour le groupe dans son ensemble ${ }^{7}$. Radcliffe-Brown étend cette analyse, élaborée pour comprendre les fonctions des relations à plaisanteries au sein du groupe de parenté, aux rapports entre groupes claniques et ethniques: de telles pratiques permettent de faire ressortir par intermittence, sur le mode pacifique car non sérieux de la plaisanterie, les tensions qui naissent du contact entre groupes étrangers. On retrouve au cœur de la théorie fonctionnaliste l'idée que les relations à plaisanteries sont des modes de gestion des tensions par délivrance cathartique. Le souci de Marcel Griaule (1948), dans son article "L'alliance cathartique» est d'invalider les réflexions de Radcliffe-Brown, selon lui trop axées sur le système de parenté. Il défend au contraire l'idée que les relations à plaisanteries, institution sociale par excellence, ne prennent sens que dans leur confrontation aux croyances cosmologiques centrales dans une culture donnée, en particulier aux théories locales de la personne et aux récits d'origine des groupes en relation. À partir du cas des Bozo et des Dogon du Mali, Marcel Griaule met en évidence les éléments du système mythicoreligieux qui rendent compte des relations à plaisanteries appelées mangou liant ces deux groupes. Les Bozo et les Dogon, apparus ensemble au moment de la création du monde, auraient prononcé un serment solennel sur une enclume, «disant, entre autre, que les deux peuples sont mangou, réciproquement, puisqu'ils étaient ensemble sur l'édifice céleste » (Griaule $1948: 249$ ), et validant les pouvoirs réciproques qu'ils ont de se purifier par l'échange d'injures ou de plaisanteries au quotidien et l'accomplissement de services rituels.

12 Ces deux types d'approches théoriques vont faire des émules. En Tanzanie, J. B. Christensen affiche clairement ses références à la théorie fonctionnaliste dans un article paru en 1963 sur les populations waluguru. L'auteur cherche à montrer comment, dans cette société de la ceinture matrilinéaire d'Afrique de l'Est, les relations à plaisanteries sont caractérisées par leur fonctionnalité dans la reproduction de l'organisation politique et sociale. Il considère que de telles pratiques atténuent les conflits potentiels et entretiennent l'harmonie entre groupes en contact ${ }^{8}$. De son côté, T. O. Beidelman (1966, 1997) inscrit son travail sur les populations wakaguru du nord-est de la Tanzanie dans une perspective symbolique. Il présente un panorama des croyances religieuses et des représentations symboliques relatives à la notion de pollution du corps pour faire du partenaire à plaisanteries un intermédiaire entre sacré et profane, justifiant ainsi les tâches de purification par la plaisanterie qui lui échoient.

13 Malgré les points de divergence dans les positions théoriques qui fondent le fonctionnalisme et l'analyse symbolique, ces deux approches ont tout d'abord ceci en commun qu'elles reposent sur une conception normative de la société, selon laquelle ce sont les règles - qu'elles concernent le domaine politique ou le champ religieux - qui permettent de penser le fonctionnement d'une société. Autrement dit, au lieu d'être appréhendées comme des modèles théoriques reconstruits par le chercheur après coup, les normes sociales sont considérées comme le moteur des actions individuelles, agissant de façon coercitive sur les pratiques individuelles. En second lieu, ces deux approches promeuvent un schème essentialiste qui fait des groupes sociaux des entités homogènes aux caractéristiques immuables, et oublient d'interroger les frontières des groupes, la 
labilité des ethnonymes, la pluralité des appartenances, la construction des groupes. Une troisième critique qui peut être formulée est que ces courants théoriques se caractérisent par un déni de l'histoire : aucune des études jusqu'à présent citées ne s'interroge sur les changements, les évolutions, les déplacements qui affectent les pratiques dites relations à plaisanteries. Enfin, elles insistent sur le bon fonctionnement du groupe (que ce soient le clan, la caste ou l'ethnie), sur la reproduction des modes d'organisation et des représentations qui le fondent, passant sous silence les dysfonctionnements, la conflictualité et les stratégies de légitimation du pouvoir. La société est perçue comme une entité homogène, sans clivages entre groupes, sans points de rupture ni heurts entre générations, entre hommes et femmes, entre regroupements sociaux autour d'intérêts propres. Dans cette optique, les relations à plaisanteries ne seraient donc rien de moins qu'une « coutume » ou une « tradition » dans le sens classique du terme, c'est-à-dire une institution transmise telle quelle au cours du temps sans jamais subir d'inflexions.

14 À l'évidence, l'accent porté avec insistance sur la dimension comique et/ou cathartique des relations à plaisanteries témoigne d'un désintérêt pour sa dimension politique. Défendre l'idée que le comique est l'essence même de ces relations, et qu'il constitue un lieu de défoulement des tensions psychologiques et sociales, évacue tout questionnement sur les rapports de force et les enjeux identitaires qui les sous-tendent. Le second point qui a fait couler beaucoup d'encre, celui de la grossièreté ou de l'obscénité, renvoie aussi au refus de s'attacher à une analyse des enjeux de pouvoir qui rendent compte des pratiques sociales. En effet, de nombreux auteurs ont insisté sur cette dimension des relations à plaisanteries, l'interprétant comme l'expression de traditions bizarres, si ce n'est barbares, signes d'un monde non « civilisé » auquel manquerait le savoir-vivre censé caractériser le $\mathrm{XIX}^{\mathrm{e}}$ siècle européen ${ }^{9}$. Cette complaisance pour le vulgaire se superpose à la solution de facilité qui consiste à faire du plus manifeste l'enjeu central des «coutumes » indigènes. Toutefois, plutôt que d'attribuer l'obscénité à une essence proprement africaine, incarnation de la sauvagerie, il convient d'en saisir les logiques d'émergence et de déterminer les enjeux qui rendent compte de l'usage d'un langage et de comportements de transgression.

Les conditions opératoires des « relations à plaisanteries » :

enjeux de pouvoir et affirmation identitaire

Un retour historique sur les rapports entretenus par les unités politiques précoloniales peut permettre de prendre de la distance avec la catégorie savante de relations à plaisanteries. Au lieu de se demander quelle fonction remplissent les pratiques ici étudiées, évacuant par-là même toute réflexion historique et politique, il convient de reconstituer les logiques qui président à leur émergence et à leur reproduction (Fouéré 2004a). Les trois dimensions majeures de la définition classique des relations à plaisanteries, à savoir qu'elles lient des groupes ethniques considérés comme des entités homogènes, qu'elles sont le résultat d'alliances entre ces groupes, et qu'elles donnent naissance à des relations égalitaires, sont soumises à un examen critique à partir des données disponibles sur l'histoire de la Tanzanie.

Chevauchements constants des sociétés à l'époque précoloniale

16 À l'époque précoloniale, dans tout le territoire de la Tanzanie actuelle, les groupes politiques pertinents, ou ce qu'on peut appeler les niveaux d'agrégation réels, peuvent se placer sur un continuum allant des unités socio-économiques dominées aux grands royaumes dominants. Ces petites unités et ces royaumes n'avaient pas de frontières immuables. Des regroupements de tailles diverses s'opéraient en fonction des 
conjonctures politiques, incluant et rejetant au gré de leur élargissement ou de leur contraction des populations politiquement ou économiquement plus faibles (Iliffe 1969, 1979). La labilité des frontières allait de pair avec des variations constantes des ethnonymes. Les appellations étaient largement indexicales, c'est-à-dire fonction des interlocuteurs, de la situation dans laquelle ils se trouvaient, de leur espace géographique de résidence, etc. (Brandström 1986 ; Iliffe 1979). Au XIX ${ }^{e}$ siècle, des grandes organisations sociopolitiques centralisées ont émergé à l'intérieur du territoire, tels l'« État » nyamwezi et l'«État» sukuma. Ces nouveaux pôles politiques acquièrent leur pouvoir par la détention de rôles prépondérants dans le commerce caravanier de longue distance (Kjekshus 1977; Koponen 1988). Ces pouvoirs politiques organisés exerçaient une forte pression sur les sociétés d'agriculteurs situées à leurs périphéries. L'historien Andrew Roberts $(1970: 70)$ rappelle que celles-ci les pourvoyaient en biens de première nécessité : "Chiefs obtained a special share of their subjects' labour and produce : and not only the harvest but animal and mineral welter.» Ce que le processus de formation étatique qui prend de l'ampleur au XIX ${ }^{e}$ siècle va finalement induire, c'est une distinction croissante entre sociétés dominantes, ayant accès aux ressources économiques et sociales, et sociétés dominées, à l'unité politique faible. Les premières imposaient aux secondes certains types de relations, fondées sur la dépendance économique («relations tributaires $»)$ ou sur la prédation active («relations prédatrices $»)^{10}$. Ces situations n'étaient toutefois pas figées: elles suivaient les phénomènes d'édification et de renforcement, ou au contraire de fragilisation et de contractions des organisations politiques centralisées (Amselle \& M'Bokolo 1985). C'est dans ce contexte de rapports de pouvoir inégalitaires entre unités politiques que se sont construites des identités de plus en plus spécifiées, qu'atteste la fixation croissante des ethnonymes.

Les reconstructions historiques qui ont mis en évidence la fluidité des modes d'organisation politique, l'absence de supra-organisation de type ethnique et les remaniements incessants des catégories identitaires jusqu'au début $\mathrm{du} \mathrm{xx}^{\mathrm{e}}$ siècle laissent clairement apparaître qu'on ne peut pas se référer aux schèmes explicatifs classiques des relations d'utani. Ainsi, dire : « tel groupe ethnique a des relations à plaisanteries avec tel autre groupe ethnique» illustre les processus de réappropriation des ethnonymes à l'époque coloniale, mais passe sous silence le remodelage continu des frontières des groupes qui caractérisait la période précoloniale. Autrement dit, la définition ethnographique des relations à plaisanteries, qui repose sur l'appréhension des groupes comme entités politiques, culturelles, linguistiques et identitaires, aux contours bien définis, bref comme des « ethnies », est démentie par les données historiques.

Des relations à plaisanteries à géométrie variable

Le deuxième point à interroger dans la définition classique de notre objet d'étude renvoie à ses origines. Selon les récits locaux évoqués par les théoriciens de l'utani, les situations de contact entre populations se seraient accompagnées de l'établissement d'accords de non-agression ou d'entraide (via des échanges commerciaux, matrimoniaux, et de services). L'objectif explicite de ces accords était de résoudre la conflictualité inhérente à toute rencontre entre groupes étrangers, voire les conflits réels qui éclataient, mais aussi d'institutionnaliser les relations informelles qui pouvaient se développer au cours du temps. Depuis les études de Malinowski (1922) sur la kula et celles de Boas (1898) sur le potlatch, on n'ignore pas l'importance des prestations de biens et de services dans l'entretien des relations pacifiques entre groupes. La circularité des dons et des contredons, dont Marcel Mauss (1924) a rendu compte, et qui est un thème maintes fois repris 
dans la littérature ethnologique, est une caractéristique majeure des relations sociales. Le développement des relations d'utani constituerait une seconde étape dans l'officialisation des liens entre populations. En effet, d'après les récits locaux qui alimentent les réflexions des ethnologues précédents, des règles de comportement auraient été édictées pour confirmer ces conventions de non-agression et/ou d'échanges pacifiques, instituant les pratiques de relations à plaisanteries. L'hypothèse centrale qui se dégage donc des écrits ethnologiques passés est que les relations à plaisanteries ne seraient pas tant une alliance en elles-mêmes qu'une conséquence des alliances politiques, économiques ou matrimoniales. Dans cette perspective, on peut dire que les échanges de biens et de services entre groupes distincts entrant dans la catégorie utani relèvent des pratiques universelles de connections des groupes et d'entretien des relations tissées.

Toutefois, la première erreur des auteurs classiques est de concevoir ces alliances politiques et/ou économiques comme des contrats infrangibles qui auraient perduré au fil du temps sans être jamais remis en question. Sachant que les regroupements sociopolitiques étaient des entités mouvantes, il est certain que ces alliances étaient sujettes à de grandes fluctuations. Des groupes pouvaient, dans une conjoncture politique et économique donnée, passer des compromis, qui duraient jusqu'à ce qu'un nouveau contexte et des nouveaux intérêts émergent. Les anciennes alliances étaient alors aménagées ou simplement détruites, tandis que d'autres alliances étaient scellées avec de nouveaux groupes. Puisque les alliances politiques, économiques et matrimoniales n'étaient pas des pactes éternels, les pratiques d'utani ne l'étaient pas non plus. Produits de ces alliances, elles ne pouvaient que suivre les processus de décomposition et de recomposition des conventions entre populations. Cela expliquerait la multitude des relations à plaisanteries qui ont été répertoriées sur le territoire tanzanien et l'impossibilité de déterminer leur "origine», c'est-à-dire l'époque et le lieu de leur émergence.

20 La seconde réserve à formuler à l'encontre des théories ethnologiques classiques concerne les modes concrets de production historique des relations à plaisanteries. Selon leurs tenants, les relations à plaisanteries seraient le résultat d'une décision unilatérale des chefs et de la soumission des populations. Or une telle vision des « origines » de ces relations ne concorde pas avec les données historiques sur la Tanzanie précoloniale. En effet, comme on a pu le voir dans les développements précédents, les groupes locaux constituaient rarement des unités politiques fortement centralisées. Ceux qu'on appelait les « chefs » n'étaient en rien des décideurs tout-puissants capables d'imposer par la force des clauses aussi précises que des pratiques d'entraide ou des comportements de moquerie. Dans le cas spécifique des attitudes dites « de plaisanteries ", l'argument selon lequel des chefs auraient institué de la grossièreté obligatoire, décidant en outre que des populations devraient se voler des objets pour les restituer contre une petite rançon ou se coucher dans la tombe d'un mort, relève de la fiction plutôt que de la réalité historique. Par conséquent, il faut plutôt envisager une institutionnalisation a posteriori des pratiques quotidiennes émergeant des frictions et des heurts entre populations qu'une politique volontariste de déploiement des relations à plaisanteries comme outil diplomatique.

21 Ainsi, en couplant les informations disponibles sur les modes d'organisation sociale des populations de Tanzanie, on est amené à affirmer que les pratiques aujourd'hui désignées par l'appellation utani doivent être conçues comme des compromis «locaux » et "fluctuants" entre des groupes eux-mêmes "mobiles» (dans un sens géographique, mais aussi morphologique). Elles sont bien plus liées aux situations de contact conflictuel 
entre membres de groupes étrangers, situations où transparaissent des rapports de force variables, qu'elles ne sont le résultat de la passation d'alliances codifiées. En outre, les contractants n'étaient pas sur un pied d'égalité. C'est en effet à la suite de stratégies, mises en place soit par le plus faible dans le but de n'être pas entièrement assimilé ou détruit, soit par le plus fort pour légitimer sa prise de pouvoir sur des dépendants, que de tels pactes ont pu prendre forme. On peut donc dire que les relations à plaisanteries sont déterminées par la construction politique des groupes sur une aire géographique aux contours flous. Elles sanctionnent l'état des relations inégalitaires à un moment donné dans un espace donné.

Production et reproduction des dissymétries de pouvoir

Dans son travail sur les castes d'Afrique de l'Ouest, l'ethnologue et historienne Tal Tamari (1997) défend une hypothèse analogue à la nôtre, à savoir que les alliances à plaisanteries auraient transformé des rapports de force entre les groupes locaux en des relations spécifiques ayant pour but de maintenir les groupes dans leurs positions respectives. Ces alliances auraient constitué un "instrument de la diplomatie mandingue », c'est-à-dire un instrument de règlement des relations entre groupes. Mais l'auteur a le tort de concevoir ces alliances comme des compromis stables, éternellement valides après avoir été énoncés. De plus, en posant les alliances à plaisanteries dans une antériorité historique par rapport à l'institution des castes, puisqu'elle considère que ce sont les alliances à plaisanteries qui ont servi de modèle à la fondation des castes, elle passe à côté de l'idée que ces deux phénomènes doivent être pensés dans leur concomitance: les supposées alliances à plaisanteries, parce qu'elles nécessitent la désignation des contractants, leur donnent en même temps consistance. Elles sont donc en quelque sorte "performatives ", faisant advenir les groupes en même temps qu'elles les lient. Comme le rappelle Michel Foucault (1997), les rapports de pouvoir ne se mettent pas en place entre des groupes, ils créent ces groupes; ils viennent les faire exister ${ }^{11}$. Il semble bien que l'erreur courante ait été de penser notre objet d'étude présent à partir de groupes préconstruits, ou construits pour la cause, au lieu de tenter de saisir les logiques principales d'interaction et de montrer comment ces logiques déterminent au contraire ces groupes.

En nous appuyant sur les rappels historiques précédents, on est amené à faire l'hypothèse que les relations d'utani n'ont pas aboli l'asymétrie des rapports de pouvoir qui les fondent. Elles ont au contraire permis de reproduire ces rapports inégalitaires en maintenant les groupes dans leurs positions respectives. Cette thèse est soutenue par l'ethnologue Stephen Lucas (1974) dans ces travaux sur l'utani au Tanganyika. Prenant le contre-pied des analyses structuralo-fonctionnalistes, il défend l'idée que les relations d' utani reposent sur les rapports de force passés et constituent un cadre dans lequel sont réaffirmées, et donc justifiées, les dissymétries actuelles dans la détention du pouvoir ${ }^{12}$. Les réflexions développées par Michel Foucault (1997) permettent d'appuyer cette thèse. Ses travaux sur le politique attestent de la reproduction constante des rapports sociaux et de leur détermination par les rapports de force passés établis entre les belligérants dans les moments de guerre ${ }^{13}$. Lorsque le "pouvoir politique", c'est-à-dire les groupes dominants ayant autorité sur un ensemble d'individus, met fin aux guerres, il ne se donne pas pour objectif de délier les différents nœuds autour desquels les rapports de force ont pris consistance. Au contraire, le pouvoir politique réinscrit « perpétuellement ce rapport de force, par une sorte de guerre silencieuse, et [il] le réinscrit dans les institutions, dans les inégalités économiques, dans le langage, jusque dans les corps des uns et des autres » 
(Foucault 1997: 16). Opérant un retournement de la proposition de Clausewitz (1832), pour dire que «la politique, c'est la guerre continuée par d'autres moyens", Foucault (1997 : 41) propose un nouvel axe d'analyse permettant de penser la perpétuation du fonctionnement inégalitaire des rapports sociaux. Cette idée que le pouvoir politique réinscrit les rapports de force passés dans les différents domaines de la vie sociale est particulièrement pertinente pour saisir la production et la reproduction des relations à plaisanteries.

Les relations à plaisanteries comme micro-pratiques de pouvoir

L'analyse des situations et des pratiques auxquelles les acteurs locaux appliquent aujourd'hui le signifiant « relations à plaisanteries » permet de rendre compte des enjeux sous-jacents qui les animent. Ces enjeux, toujours locaux, s'inscrivent dans deux grandes thématiques: l'identité et le pouvoir. En premier lieu, l'expression de relations à plaisanteries est appliquée à des pratiques qui fonctionnent comme des marqueurs des frontières des groupes. Elles participent donc de la production in situ des identités que les acteurs décident de mettre en avant. En second lieu, ce référent désigne des pratiques qui réactualisent les enjeux de pouvoir passés ou dévoilent des enjeux présents pour imposer au cadre de l'interaction une certaine inflexion, placée sous le signe de rapports dissymétriques de pouvoir. Ces deux thématiques doivent être envisagées comme les deux faces d'un même phénomène de positionnement des individus et des groupes dans l'espace social. Toute affirmation identitaire renvoie à la question du pouvoir, comme toute recherche d'assise du pouvoir prend, parmi d'autres stratégies, la voie d'un renforcement de l'identité.

Les exemples qui suivent sont tirés d'un terrain mené au cours de l'année 2001-2002 en Tanzanie, dans la ville de Morogoro. Bien que rejetant la vision primitiviste selon laquelle le site privilégié de la recherche serait un petit village coupé des néfastes influences occidentales ${ }^{14}, \mathrm{j}$ 'ai organisé et vécu mon séjour sur un mode classique de coupure spatiale et d'immersion dans un milieu étranger. Résidant dans une maison où logeaient plusieurs ménages, ayant tissé des liens avec quelques familles du voisinage, j'ai pu être le témoin d'interactions qualifiées d'utani par des interlocuteurs ${ }^{15}$ qui étaient pour moi des familiers ${ }^{16}$.

Les mises en scène de l'identité

L'observation des situations d'interaction montre que les relations à plaisanteries puisent dans les référents identitaires classiques de l'espace social dans lequel elles sont mises en œuvre, en même temps qu'elles constituent une modalité des positionnements identitaires individuels et collectifs. Contre l'idée que les identités seraient des entités figées, transmises telles quelles au cours d'une histoire sans histoire, on constate qu'elles se caractérisent par des assignations au présent qui les produisent et les reproduisent à chaque rencontre entre locuteurs.

En Tanzanie, les référents identitaires ethniques et claniques sont quotidiennement utilisés par les acteurs locaux comme des catégories pertinentes d'identification. En situation d'utani, ces référents s'accompagnent de la formulation de stéréotypes relatifs aux traits culturels, religieux, sociaux, etc. qui sont censés typifier un groupe ethnique donné. Il est à noter que ces traits sont évoqués sur un mode substantialiste, c'est-à-dire qu'ils sont perçus comme des caractéristiques intrinsèques à un groupe pensé comme totalité homogène (Fay 1995). Cette substantialisation des identités à l'œuvre dans les 
énonciations entre partenaires à plaisanteries apparait clairement dans les exemples suivants :

- Une femme plaisantait avec un homme en lui disant : «Je suis mnyamwezi. Parlons kinyamwezi ensemble » ${ }^{17}$, à quoi l'homme a répondu, sans rire : « Les Wanyamwezi ne savent pas parler/ne parlent pas » ${ }^{18}$. La femme a alors répliqué : « Et bien toi, je vois que tu aimes l'utani !» ${ }^{19}$.

- En Tanzanie, on entend souvent dire que les Wagogo sont des mangeurs de viande et peuvent se contenter de se nourrir de cet aliment. Une femme mgogo cuisinait très régulièrement de la viande, et en grande quantité. Les autres femmes de la maisonnée plaisantaient souvent sur son grand appétit. Au retour d'un voyage dans son village natal, elle avait ramené de la viande de buffle séchée, dont elle avait revendu une part aux autres femmes, mais dont elle s'était octroyé un large morceau. Cela avait donné lieu à de nouvelles plaisanteries sur l'amour de la viande chez les Wagogo. Le même cliché sur les Wagogo a été attesté au cours d'une soirée chez des amis. Une invitée avait refusé de rester pour dîner, en prétextant que dans cette maison, il n'y avait jamais de viande au repas. Un des hôtes avait répliqué que les Wagogo posaient toujours problème dans les dîners car ils n'aimaient que la viande.

- Un père de famille m'avait présenté un de ses apprentis, en précisant qu'il était originaire de la région de Tanga, au nord-est de la Tanzanie. Il avait ajouté que les gens de Tanga étaient des fourbes et des hypocrites qui aimaient raconter des mensonges. Ni le père de famille ni son apprenti ne riaient pendant l'énonciation de ces paroles. Mais sa femme, qui nous accompagnait, m'avait regardée en riant, et avait précisé que son mari aimait plaisanter avec son apprenti : «Il aime "faire utani" avec lui » ${ }^{20}$.

Ces exemples montrent que les catégories identitaires sont reprises par les individus dans les échanges d'utani. Elles le sont à la fois comme justificatif de l'utani (les gens rient de la femme qui aime la viande parce qu'elle est mgogo; le père de famille se moque de son apprenti parce qu'il est de la région de Tanga) et comme contenu de l'utani (les gens prennent pour cible le groupe d'appartenance de leur interlocuteur dans sa totalité, et insistent sur les traits qu'ils considèrent être les plus saillants de ce groupe ethnique). Le marqueur identitaire fournit un cadre à l'énonciation et à l'adoption de certains comportements, en même temps qu'il en est la ressource.

Quand des individus invoquent la catégorie identitaire qu'ils tiennent pour la leur ou pour celle de leurs interlocuteurs, cela pour justifier les pratiques d'utani, ils affirment leur pertinence. Dire : «Toi, le Mgogo, tu aimes la viande », c'est valider les ethnonymes existants et les stéréotypes auxquels ils sont liés. Mais c'est aussi assigner une nouvelle fois à l'autre sa place dans le réseau des identités disponibles. C'est lui dire «toi, tu appartiens à tel groupe (puisque je t'appelle ainsi) ", groupe dont l'ethnonyme suffirait à attester de l'existence. La situation suivante met en évidence le lien qui existe entre énoncer une identité et l'assigner.

- Un jeune vendeur mgogo avait désigné un de ses amis en me disant qu'il était son mtani, puisqu'il était msukuma. Il pouvait donc l'embêter sans qu'il puisse réagir. Ce faisant, il lui a arraché le torchon que son ami tenait au-dessus de la tête pour se protéger de la pluie qui commençait à tomber. Lorsque j'ai demandé à ce dernier s'il était d'accord pour dire que les Wagogo étaient ses watani, il a répliqué : « Lui, il n'est pas mon mtani, ils travaillent dans nos champs, nous sommes leurs patrons $"{ }^{21}$.

Cette situation est l'exemple d'une assignation identitaire externe: un individu « rappelle » à son ami son identité ethnique (msukuma), mais ce faisant, il la pose comme 
quelque chose d'irréfutable. L'autre accepte cette assignation, affirmant par ses propos qu'il se reconnaît bien de l'identité assignée. Les processus par lesquels s'opèrent les "positionnements sociosubjectifs» des acteurs, selon la terminologie employée par Cécile Canut (2002), c'est-à-dire la manière dont les individus se placent individuellement sur l'échiquier des identités sociales et se « ressentent » d'une identité donnée, sont ici visibles : assignation externe, acceptation de cette assignation, utani (saisie du torchon), passage d'une interaction entre deux individus (lui, il est, mon) à une problématique qui met en jeu les groupes (ils travaillent, nos champs, nous sommes, leurs patrons). Dans le cas présent, les individus tendent à réaffirmer des positions et des statuts pré-assignés, plutôt qu'ils ne les remettent en question. On est dans une logique de reproduction des identités.

31 Les analyses contextuelles des situations d'assignation du terme utani, présentées cidessus, s'inscrivent dans les conceptions dites pragmatiques de la langue. Contre l'illusion instrumentaliste selon laquelle le langage se réduit à un moyen matériel de communication du savoir, ou de transmission de l'information, la pragmatique comme science des usages sociaux et contextuels de la langue défend l'idée que la signification d'un énoncé ne se donne pas en soi mais dans le rapport qu'il entretient avec des éléments extérieurs à lui-même ${ }^{22}$. Pour reprendre le vocabulaire de la sémiotique et de la pragmatique, on peut dire que la fonction dénotative ${ }^{23}$ de la langue, ne constitue qu'un type parmi d'autres des «jeux de langage» (Wittgenstein 1985) ${ }^{24}$ selon lesquels les significations d'un énoncé se modulent en fonction de la position des locuteurs et de la situation d'énonciation. Dans cette perspective, c'est avant tout la mise en lumière de la caractéristique du langage comme mode d'action pratique - caractéristique qui apparaît dans les travaux de sémiotique sous le terme de «fonction performative» du langage, maintes fois discutée depuis l'ouvrage célèbre de J. Austin (1962) ${ }^{25}$ - qui a contribué aux développements des réflexions sur les usages sociaux de la langue et qui peut servir de référence à une pragmatique des relations à plaisanteries. Certes, comme l'a souligné P. Bourdieu (1980), il ne suffit pas toujours de dire pour que cela soit. Le statut du locuteur ainsi que le contexte de l'énonciation sont les conditions majeures de l'efficacité de la parole. Mais ce qu'on voit se dessiner de spécifique avec les relations à plaisanteries, c'est que tout individu, parce qu'il est toujours le détenteur et le défenseur d'une certaine identité dans le jeu social, est en mesure de produire, par ses pratiques langagières, ce qu'il dit ou fait. Cette assignation externe peut être acceptée, mais aussi refusée, rejetée, contournée, de sorte que c'est bien dans les situations présentes que les individus opèrent des choix d'identification. Autrement dit, les identités sont l'objet d'un combat implicite sur le mode langagier : elles sont soumises à une agonistique générale.

Dire que l'identité est produite au moment même où elle est énoncée ne revient pas à défendre la thèse d'un surgissement identitaire spontané. Les individus opèrent des choix parmi les catégories sociales disponibles dans un espace donné, et ces choix sont orientés par des effets de structure irréductibles à la seule situation présente. Pour le dire autrement, les positionnements identitaires individuels ne peuvent être coupés des catégories sociales pertinentes que les individus connaissent. Il s'agit donc bien de positionnements "sociosubjectifs", parce qu'ils sont à la fois orientés par la structure sociale et les rapports de pouvoir qui la traversent, et mis en œuvre au niveau interindividuel selon des stratégies subjectivement énonçables. L'ethnologue Stephen Lucas (1974: 7) avait déjà noté l'entrecroisement permanent entre individuel et collectif dans le cas des pratiques d'utani, le passage constant qui s'opère entre le niveau 
interindividuel et le niveau interethnique. Il affirme en effet que, dans les situations qualifiées d'utani, "the interaction rarely remains at the individual-to-individual level and it almost invariably results in a defence of one's group and a heightened sense of solidarity in opposition to the opponent's group ». Les identités individuelles sont donc des élaborations contemporaines, qui puisent dans un fond identitaire commun pour s'afficher et affirmer leur inscription dans un ensemble qui les englobe.

Durcissements ou franchissements des frontières entre groupes

Il est possible d'élargir nos propos en montrant que les relations à plaisanteries ont pour enjeu central la construction des groupes sociaux. En effet, le fait que les clichés, les pratiques culturelles, religieuses, sociales, etc., considérés comme caractéristiques d'un groupe, soient justement le contenu même des énoncés de relations à plaisanteries, dans leur dimension de moquerie, incite à penser que ce sont les frontières des groupes qui sont en jeu. Cette hypothèse s'appuie sur les analyses de la production de l'altérité, développées dès la fin des années 1960, et qui semblent incontournables si l'on veut saisir les logiques des pratiques couramment qualifiées de relations à plaisanteries. Comme le rappelle Fredrick Barth (1969: 15) dans l'introduction à son ouvrage collectif sur les frontières entre groupes ethniques, « entailed in ethnic boundary maintenance are also situations of social contact between persons of different cultures : ethnic groups only persist as significant units if they imply marked differences in behaviour, i.e. in persisting cultural differences ». Autrement dit, c'est par l'affirmation de la différence que sont construites et maintenues les frontières sociales. Et c'est justement cette opération de maintien et de construction renouvelée de l'identité, qui transparaît dans les situations de contact social où sont mis en œuvre les gestes et les paroles de relations à plaisanteries. Interagir à partir des critères d'appartenance (c'est parce que l'individu se reconnait de tel groupe ethnique qu'il "plaisante» avec un individu de tel groupe ethnique), et faire surgir les caractéristiques culturelles, sociales, religieuses, économiques, culinaires, etc. attachées à ces catégories, c'est redessiner dans l'interaction ce qui sépare de l'autre; en d'autres termes, c'est signaler à nouveau la frontière entre membres et non-membres, entre autochtones et étrangers.

Dans un article qui s'inscrit dans cette orientation constructiviste des groupes sociaux, Per Brandström (1986) indique qu'en Tanzanie, la construction des identités nyamwezi et sukuma a été concomitante de la stéréotypisation des traits culturels et linguistiques « in a form of stories people tell about each other. The easterners, for example, in a jokingly manner, are said to be outspoken, quarrelsome and cunning people, while at the same time, stories are told about how timid and gullible the southerners are ». C'est de ce même phénomène qu'attestent les pratiques d'utani: reproduire les stéréotypes, c'est opérer des différenciations entre «eux» et "nous». Et il est vrai que malgré le franchissement constant de ces frontières, les remaniements et les négociations identitaires qui sont visibles dans les interactions au quotidien, une certaine ligne de démarcation se stabilise régulièrement (Kopytoff 1987), d'autant plus évidente aujourd'hui qu'elle a été figée par l'écrit, principalement celui des Européens.

L'utani, signifiant qui englobe les pratiques par lesquelles sont affirmées ou infirmées les frontières entre les groupes de Tanzanie, fonctionne comme un mode de catégorisation de l'autre, de l'étranger. Or, il est intéressant de voir que ce même caractère a été mis en évidence dans d'autres régions du monde. Ainsi, dans un article sur la notion d'étranger dans le monde mandingue, J.-L. Amselle (1996b) montre que la catégorie de senankuya, qui renvoie à un pacte d'alliance et de sujétion entre des formations politiques du type de la 
chefferie au Mali, produit l'autre en énonçant ce qui nous en distingue: les pactes d'alliance s'accompagnent en effet d'interdits sexuels, matrimoniaux et tactiles qui rappellent que des relations spécifiques se mettent en place avec cet autre groupe. L'extériorité, ou l'altérité, est une construction que les rapports de senankuya viennent valider et reproduire. De même, dans son article sur les relations de senankuya au Mali, C. Canut (2002) rapporte des cas de moqueries qui portent sur les caractéristiques sociales et culturelles attachées aux groupes d'appartenance des personnes impliquées. L'auteur poursuit son analyse en soulignant que les termes par lesquels sont désignées les identités constituent des «signifiants vides » qui prennent sens dans leur opposition à d'autres identités. Les pratiques de senankuya jouent de cette opposition en rappelant les modalités de l'appartenance et de la non-appartenance. Certes, on peut avoir l'impression que les relations à plaisanteries, en tant qu'énonciation de stéréotypes, figent les groupes ethniques des interlocuteurs en entités absolument distinctes. Mais sachant que ces stéréotypes sont l'objet d'un affrontement entre interlocuteurs, il est plus juste de considérer ce type d'énoncés comme le lieu où les tensions entre identités se donnent à voir.

36 Cette qualité propre aux pratiques d'utani qui consiste à marquer la différence entre « eux » et « nous » explique pourquoi il arrive que des « étrangers ", c'est-à-dire des nonTanzaniens, soient la cible de plaisanteries. Ainsi, mon séjour à Morogoro a été propice à l'énonciation d'utani à mon encontre. Mama S., que je considérais comme une amie dans la mesure où il me semblait pouvoir aborder des sujets plus personnels avec elle, « faisait utani » avec moi fréquemment.

- Un jour, elle m'a affirmé qu'elle avait cuisiné les bagia (petites croquettes de farine de haricots secs épicées) que nous avions pris au petit-déjeuner. Dans l'après-midi, nous sommes passées près d'un stand de bagia, qu'elle m'a montré en me disant : « Je t'ai fait marcher, les bagia, je les ai achetées ici. » Je lui ai fait remarqué qu'elle aimait bien me faire marcher, à quoi elle a répliqué : « Je suis ton $m$ tani ${ }^{26}$.

- Un soir, Mama S. et moi avons entamé une discussion sur le fait que beaucoup de personnes dans la maison où nous habitions ne savaient pas plaisanter. Elle avait alors affirmé que faire des plaisanteries était quelque chose de sympathique, et qu'elle était contente que j'aime plaisanter. À ce moment, elle a voulu me faire croire qu'il fallait que je cède mon lit pour la nuit à la propriétaire de la maison, venue de Dar es Salaam récupérer les loyers. Cela signifiait qu'il fallait que je dorme par terre, sur une natte. Me doutant qu'elle faisait une plaisanterie, je lui ai demandé si je pouvais dormir à l'intérieur ou s'il fallait en plus que je reste dehors. Elle a répondu que je pouvais dormir dans la chambre, puis a éclaté de rire avant d'expliquer qu'elle me « faisait marcher ».

- Après un diagnostic de paludisme, j'avais acheté des médicaments dont la notice indiquait qu'il était déconseillé de manger des nourritures contenant des lipides pendant quelques jours. J'avais dit aux femmes de la maison que je ne pouvais manger que du pain, sinon les médicaments allaient me tuer. Mama $S$. avait répliqué qu'elles toutes viendraient à mon enterrement, qu'elles feraient utani, et qu'elles diraient : « Qui va nous payer des bières maintenant?»

La mise en place de relations à plaisanteries par confrontation quotidienne participe d'une dynamique d'inclusion d'un étranger dans le tissu social et symbolique, en même temps qu'elle permet de rappeler les relations de pouvoir entre interlocuteurs, de les reproduire ou de les renverser. Par ses paroles, Mama S. propose en effet une hiérarchie sociale dans laquelle je suis en position d'infériorité par rapport à la propriétaire de la 
maison (je dois céder mon lit); mais elle renvoie aussi aux différences de pouvoir économique qui nous séparent (c'est moi qui offre les bières). Les dissymétries de pouvoir sont soulignées pour mieux s'en moquer, et les moqueries réciproques désactivent l'étanchéité des frontières entre autochtones et étrangers. On voit donc combien les relations à plaisanteries sont labiles et s'adaptent au contexte de leur production. En ce sens, on est bien loin du modèle juridique qui a nié de telles caractéristiques.

Production et reproduction des rapports de force

La réactualisation des frontières des groupes, c'est-à-dire l'assignation de l'interlocuteur dans une catégorie donnée, permet de reproduire les schèmes de pouvoir passés pour les adapter à la situation présente. La question identitaire ne peut être abordée sans que soit mise en lumière la manière dont elle s'imbrique avec la question du pouvoir. On constate en effet que dans le cadre des relations à plaisanteries, les individus jouent avec les modes de relations hiérarchiques passées entre les groupes ethniques et claniques, mais aussi professionnels, nationaux, etc., et les réactualisent pour définir les relations hiérarchiques contemporaines.

En Tanzanie, la référence aux rapports de pouvoir passés, historiques ou fictifs, entre les groupes ethniques est une pratique courante dans l'utani. Elle sert de fondement pour réaffirmer une position de dominant, ou pour contrecarrer les tentatives d'un des locuteurs de se poser comme dominant. Ce fait est apparent dans l'exemple du jeune vendeur mgogo. Je rappelle qu'un des vendeurs, auto-identifié comme mgogo, avait arraché un torchon des mains de son mtani Msukuma. Celui-ci avait alors répliqué qu'ils n'étaient pas watani, car les Wasukuma étaient les patrons des Wagogo. L'enjeu de cette situation est bien la détermination d'une position de dominant. D'un côté, celui qui dérobe le torchon exprime le fait que les objets de l'autre sont les siens, qu'il en est en quelque sorte le possesseur en dernière instance. L'autre est relégué dans une position subalterne caractérisée par la non-possession. Celui à qui l'on a dérobé le torchon exprime en retour qu'historiquement, le groupe auquel il s'identifie, celui des Wasukuma, était en position de domination sur le groupe de son interlocuteur (en l'occurrence wagogo). Il utilise donc de manière stratégique des référents historiques pour nier la pertinence du retournement de situation que son interlocuteur essaie d'opérer. L'opposition présente entre possesseur/non-possesseur prend racine dans l'opposition historique signalée entre patron/ employé.

Dans l'exemple suivant, l'interaction entre un chauffeur et sa passagère illustre la manière dont les catégories sont aussi utilisées par le chauffeur dans le but de légitimer la position dominante dans laquelle il se trouve au cours de cette rencontre.

- Un chauffeur a décidé de s'arrêter pour prendre en stop une femme qui lui faisait signe sur la route. Elle est montée à l'avant du véhicule, mais sans saluer. Le chauffeur a redémarré, puis lui a fait remarquer qu'elle n'avait pas dit bonjour. La femme a répondu : « Pourquoi tu me critiques, tu es mon mtani ? " ${ }^{27}$. Le chauffeur s'est alors enquis de son groupe ethnique, qui s'est avéré être celui des Wanyamwezi. Il lui a annoncé qu'il était mpogoro, et que par conséquent, il pouvait la critiquer, lui faire des remarques, même désobligeantes. Il est revenu sur le fait qu'elle n'avait pas salué en montant, et a précisé que c'était sans doute une façon de faire habituelle chez les Wanyamwezi ( ça, c'est leurs manières, par chez vous » ${ }^{28}$ ). Il a ri de ses paroles, tandis que la femme est restée silencieuse, mais a esquissé des sourires. L'échange a rapidement pris fin, la femme indiquant qu'elle était arrivée à destination. Le chauffeur a expliqué que la femme et lui avaient «fait utani » parce qu'elle était mnyamwezi et lui mpogoro, et qu'en outre, elle avait été malpolie. 
41 Étant donné que le chauffeur a accepté de prendre en stop la femme qui lui faisait signe sur le bord de la route, cette dernière devrait lui être redevable de son geste. Dans cette logique, elle aurait dû au moins saluer celui qui a accepté de l'aider, si ce n'est le remercier. Son silence au moment où l'interaction entre dans le cadre de l'utani (reproches du chauffeur énoncés sur le mode de la plaisanterie en référence à l'identité ethnique de sa passagère) semble signifier qu'elle accepte les remontrances de son interlocuteur, donc la position de dominée qu'il lui assigne.

Les stratégies d'assise des positions de pouvoir par référence aux caractères, réels ou fictifs, des groupes ethniques ont été mises en évidence sur d'autres terrains. Dans l'article précédemment cité sur les relations de senankuya au Mali, Cécile Canut (2002) évoque un exemple dans lequel les positions des individus sur l'échelle sociale sont rappelées aux participants de l'interaction. Un Sidibé et un Doumbia « prétendent chacun à une position dominante en réduisant l'autre à l'état d'esclave en fonction des positions sociales occupées: les Sidibé traiteront les Doumbia de "petit numu" ("forgeron"), appartenant à la catégorie des gens de castes, et à l'inverse les Doumbia insistent sur le statut d'éleveurs des Fula (Peuls) les privant de tout accès au statut de noble ». Les statuts sociaux historiquement construits, ceux de gens de caste et de nobles ${ }^{29}$, sont les catégories dans lesquelles les interlocuteurs puisent afin de réaffirmer les positions sociales présentes. Dans une même optique, Robert Launay (1977) décrit une modalité relationnelle particulière entre esclaves de seconde génération (appelés worosso) et hommes libres (horon) chez les Dioula de Côte-d'Ivoire. Les rapports entre ces deux catégories sociales se caractérisent par l'échange de plaisanteries et de propos obscènes, ainsi que par un certain nombre de devoirs d'entraide et d'assistance. L'auteur indique que ces relations, qui se nomment "tlon» en dioula, sont donc des relations à plaisanteries. Il soutient l'idée qu'elles sont le produit historique du contexte politique et économique. Elles viennent rappeler l'inégalité fondamentale des statuts sociaux d'esclaves et d'hommes libres. Ces exemples attestent du fait qu'une même dynamique agonistique travaille les pratiques d'utani, de senankuya, de tlon, etc. Par le recours à une inscription identitaire dans le corpus catégoriel de l'espace considéré, par la référence qui est faite aux rapports de pouvoir entre groupes, les relations à plaisanteries sont des pratiques de rivalité.

43 L'ensemble des situations évoquées met en lumière deux aspects entremêlés des relations à plaisanteries: des pratiques stéréotypées, des attentes sociales, des normes de la pratique; des marges de manœuvre, des stratégies, des manipulations. L'un ne va pas sans l'autre, puisque les normes, bien réelles, ne s'imposent jamais de manière coercitive. Dans le vocabulaire de Pierre Bourdieu, on peut dire que les pratiques individuelles sont orientées, mais non déterminées par les normes sociales. Certes, les stratégies ne sont pas des émergences spontanées, des créations in situ. Il y a bien un soubassement normatif qui pèse sur les agents, et que ceux-ci ne cessent de rappeler dans leurs discours sur les pratiques. Mais sur ce soubassement se greffent des pratiques dont la dimension stratégique est flagrante: les individus utilisent la rhétorique verbale et comportementale à leur disposition pour peser sur la situation, c'est-à-dire pour lui donner l'inflexion qu'ils souhaitent lui voir prendre ${ }^{30}$.

La manipulation du « cadre » : usage stratégique du référent utani

Il est important de garder à l'esprit cette propriété, déjà évoquée, que le terme utani est un signifiant flottant que les individus accolent à des situations, à des façons de faire et de dire. Autrement dit, ce sont les individus qui, par l'assignation de ce référent à une 
situation, orientent cette situation. La notion de "cadre ", telle qu'elle a été développée par Erving Goffman $(1987,1991)$, est un concept heuristique qui peut permettre de mieux cerner les modes d'usage du signifiant utani. Les cadres sont des schèmes interprétatifs dans lesquels un phénomène, un événement, une situation sont produits par un individu et grâce auxquels ils prennent sens pour un autre individu. Autrement dit, un événement est produit dans un cadre (sérieux, comique, formel, décontracté), posé au départ par un premier locuteur, et qui va permettre l'interprétation des paroles et des comportements par un auditeur. Goffman indique que toute production d'énoncés et d'actions communique des informations métacommunicationnelles ${ }^{31}$ selon lesquelles il est entendu que tel énoncé est à prendre dans tel cadre. Ces informations passent par l'intonation de la voix, les mimiques du locuteur, et un ensemble d'indices verbaux et sociaux.

Les pratiques ici étudiées sont une illustration de ces phénomènes de cadrage des interactions verbales. Le ton employé par l'interlocuteur, le recours à des normes d'attitudes et de paroles d'utani reconnues par les groupes, parfois légitimées par leur inscription dans des travaux ethnographiques, le statut ethnique et social des personnes présentes dans une situation donnée, constituent des informations qui permettent aux individus de comprendre quel est le cadre de l'interaction posé au départ. Mais le point que je tiens à souligner plus spécifiquement, c'est que l'assignation du référent utani à ce type de pratiques est un mode de cadrage particulièrement puissant. À partir du moment où le terme utani est énoncé, les participants sont assurés du cadre de l'interaction. Une telle énonciation produit le cadre ou le confirme.

Dans les exemples suivants, je présente des phénomènes de «recadrage» ou de « modulation » (dit « keying» dans les travaux de Goffman), c'est-à-dire des stratégies qui consistent à faire passer d'un cadre de production et d'interprétation des paroles émises à un autre cadre. Ce type de stratégies met en lumière combien le cadrage est une activité incontournable dans la compréhension d'une interaction.

- Mama S. était impliquée dans un conflit portant sur une somme d'argent qu'une amie lui avait confiée. Elle avait, par mégarde disait-elle, montré cet argent à la sœur de son amie, qui l'avait alors réclamé. L'amie de Mama $S$. passait souvent devant la maison où nous logions. À chaque fois, elle évoquait cette affaire de manière véhémente. Un jour, en présence de son amie, Mama S. m'a dit que cette femme était sa mtani, c'est pourquoi elle lui cherchait tant de querelles. Mais l'amie en question, ayant entendu le propos, s'est énervée et a alors affirmé haut et fort que dans le cas présent, il ne s'agissait absolument pas d'utani.

- Un exemple similaire est celui d'une querelle pour dettes entre Mama S. et un vendeur ambulant. Il semble que le vendeur avait accepté de lui vendre à crédit une lotion pour le corps. Elle tardait tant à rembourser la somme due que le vendeur passait chaque jour à la maison lui rappeler ses dettes. Mama S. rabrouait le vendeur et lui affirmait ne pas détenir l'argent nécessaire. Un jour, après une longue tirade de Mama S. sur ses faibles ressources financières, le vendeur a indiqué que la situation présente n'entrait pas dans le cadre de l' utani («Ce n'est pas de l'utani, je n'aime pas l'utani, l'utani et le commerce, ce sont des choses bien différentes ») ${ }^{32}$.

47 Ces exemples éclairent les tentatives de basculement de l'interaction dans la catégorie $d$ utani. Mais ce recadrage est un échec car il n'est pas accepté par les interlocuteurs de Mama S. En outre, on note que ceux-ci ne refusent pas le qualificatif utani sous prétexte que leur groupe ethnique d'origine ne serait pas lié à celui de Mama S. Ils le refusent pour 
la bonne raison que ce terme renvoie, pour eux, à des situations d'un autre ordre. Ils rejettent la possibilité de faire entrer les querelles pécuniaires dans la catégorie d'utani.

Il apparait donc que le référent utani est sujet à manipulations. Il n'est pas un concept univoque désignant un ensemble de paroles et de comportements bien délimités dans l'esprit des individus, toujours clairement identifiables. Certes, un ensemble d'informations liées à la situation d'interaction, en particulier l'identité des personnes en présence, le contenu sémantique des énoncés et les réactions des individus, constituent des indices sociaux utiles pour " opérer un travail de déchiffrement » (Bourdieu 1972) du cadre de l'interaction. Mais les individus peuvent user de ce terme pour indiquer aux personnes en présence quelles sont les interprétations qu'ils doivent faire de la situation, et peser ainsi sur cette situation.

La seule définition qui peut être donnée de l'utani est la suivante : le terme utani est un signifiant qui est utilisé pour désigner des situations sociales variées, plus ou moins codifiées, dans lesquelles les pratiques mises en œuvre ont pour objet conscient ou non d'affirmer un certain état du pouvoir par la manipulation des catégories identitaires disponibles dans l'espace social considéré et par la manipulation des représentations sociales qui sont associées à ces catégories. La même définition peut s'appliquer aux concepts senankuya, dakure, tlon, etc. Elle est suffisamment souple pour intégrer la variabilité et la fluidité des pratiques selon les contextes de leur émergence. On n'a donc jamais fini de "répertorier» les "relations à plaisanteries", comme ce fut le but de l'ethnologie classique, parce que les modes d'association entre ce concept et un ensemble de pratiques sociales ne cessent de se modifier en fonction du contexte historique et des situations d'interaction.

La mise en discours du concept ethnologique de « relations à plaisanteries » dans le champ politique africain

Depuis une petite dizaine d'années, la catégorie des relations à plaisanteries a fait son apparition dans les réflexions de nombreux intellectuels et hommes politiques d'Afrique. Combinées avec d'autres pratiques et valeurs traditionnelles, tels la solidarité familiale, le respect dû aux aînés ou la recherche du consensus, les relations à plaisanteries sont le plus souvent présentées comme un mécanisme de maintien de la paix sociale que le pouvoir politique en place doit promouvoir afin de préserver l'unité nationale.

Unité nationale et résolution des conflits

51 Les conflits sociopolitiques qui ont dégénéré en conflits militaires ouverts, en Guinée, en Sierra Leone, au Liberia et récemment en Côte-d'Ivoire, interrogent la capacité des pays voisins relativement stables, mais ébranlés par ces nouveaux conflits, comme le Sénégal, le Burkina Faso ou le Mali, à maintenir la paix au sein de leurs frontières. L'instabilité généralisée des pays de l'Afrique des Grands Lacs (Rwanda, Burundi, Congo-Zaïre) et les conflits militaires incessants entre factions rivales ne cessent d'alimenter les clichés sur un continent incapable d'organiser une société civile pacifique, miné par le «caractère pathologique du pouvoir» (Bayart 1989) qui s'y est développé. C'est dans ce contexte politique moderne qu'on assiste au déploiement d'un discours dont l'objectif est de présenter les pratiques de relations à plaisanteries comme des mécanismes de prévention des conflits à l'échelle de l'État-nation.

Les travaux de Amadé Badini sur le Burkina Faso, parus en 1996, illustrent l'émergence de cette préoccupation pacifiste. Présentées comme un mécanisme de régulation des conflits ethniques, "machines à fabriquer des solidarités ", les relations à plaisanteries doivent, 
selon l'auteur, être utilisées pour promouvoir la paix sociale ${ }^{33}$. Cette idée constitue le socle des réflexions des écrits successifs de nombreux intellectuels africains. Ainsi, dans une communication pour le ministère malien du Développement social, le professeur Doulaye Konaté (2001) dresse un inventaire des valeurs et des pratiques traditionnelles au Mali dans lequel la «sanankounya » se trouve en bonne place pour favoriser la tolérance, la dignité humaine et la paix civile. Le griot Bakary Soumano (2002), qui se présente comme le chef des griots du Mali, réutilise les appellations ethnologiques de "cousinage à plaisanteries", d'«alliances à plaisanteries", de "parenté à plaisanteries" et de « relations cathartiques » pour désigner les pratiques de solidarité sociale qui permettent de maintenir la paix. Au Sénégal, le juriste Abdoulaye Sakho (2002) vante les mérites de résolution des conflits par règlement à l'amiable entre partenaires à plaisanteries. On constate que les réflexions de ces intellectuels locaux ne restent pas confinées aux seules sphères des érudits africains. C'est ainsi que les sites touristiques des pays comme le Mali ou le Burkina Faso, accessibles sur Internet, présentent quelques aspects de la culture locale (populations, rites, habitudes culinaires), et ne manquent pas d'évoquer les relations à plaisanteries en indiquant leur rôle dans le développement des liens pacifiques entre les différentes composantes de la nation ${ }^{34}$. En outre, on assiste dans différentes villes du Burkina Faso à la naissance d'associations de défense et de promotion des relations à plaisanteries, telle l'Association pour la promotion de la parenté à plaisanteries de Bobo-Dioulasso (Sissao 2002). Les médias d'Afrique occidentale se font l'écho de l'intérêt porté à cette thématique ${ }^{35}$. Enfin et surtout, le Centre national de la recherche scientifique et technologique (CNRST) du Burkina Faso a indiqué dans son Plan stratégique de la recherche scientifique de 1995 que les pratiques de relations à plaisanteries devaient être une des priorités de la recherche.

Ce nouveau tournant dans l'histoire du concept de relations à plaisanteries, très frappant en Afrique occidentale, dans les pays où les pratiques de relations à plaisanteries ont toujours conservé une forte visibilité, est moins évident en Afrique orientale. Toutefois, des écrits parus au Kenya, à la fin des années 1990, attestent d'un même mouvement de défense des valeurs et des pratiques traditionnelles pour la résolution des conflits sociaux. En août 1997, le professeur de sociologie Katama Mkangi, de l'Université de Nairobi, a publié un article sur la gestion des conflits locaux qui détaille les valeurs "proprement africaines " d'égalité, de justice et de respect, et les pratiques à travers lesquelles ces valeurs se donnent à voir : l'entraide familiale, les pactes de sang et les formes de coopération entre partenaires liés par utani ${ }^{36}$. Ses travaux font suite à un article de H. Assefa (1993), sur le maintien de la paix et les formes de réconciliation au Kenya. Il est à noter qu'un programme de l'unEsco sur la place de la tradition dans les évolutions modernes, a été à l'origine de l'attention croissante portée par les intellectuels locaux à la question de la solidarité nationale.

Enfin, le développement des réflexions sur le concept de relations à plaisanteries n'est pas propre à l'Afrique. On constate en effet que de nombreux travaux de science politique, parus depuis les années 1990 en Europe et aux États-Unis, consistent à mesurer l'impact réel des relations à plaisanteries dans la résolution des conflits entre groupes ethniques en Afrique (conflict mediation ou dispute management). Les travaux de I. W. Zartman (1985, 1997) sont emblématiques de cette tendance à articuler pratiques "traditionnelles » et maintien de la paix sociale. L'auteur, déjà connu pour ses travaux sur la résolution politique des conflits socio-ethniques, a récemment fait paraitre un ouvrage dans lequel il défend une vision instrumentale des modes de faire locaux, censés être mieux adaptés, 
pour désamorcer les surgissements de violence, que les techniques diplomatiques et politiques habituellement mises en œuvre par les États, souvent sous la houlette des organisations internationales (ibid.: 2000). La contribution de Wilson-Fall (2000) sur l'usage des relations à plaisanteries par les médiateurs locaux s'inscrit dans cette logique de promotion des pratiques de conciliation par des personnages influents, mais non des officiels issus du gouvernement (Track II Policy). La tendance de ces travaux consiste même à faire équivaloir les relations à plaisanteries à des mécanismes de prévention au quotidien des conflits par les acteurs eux-mêmes (Track III Policy), permettant ainsi d'éviter le recours à des processus de médiation. Les pratiques couramment associées aux relations à plaisanteries, telles que se plaisanter et se moquer de l'autre, tout en respectant les liens d'entraide et d'hospitalité développés au cours du temps, sont perçues comme des techniques à promouvoir pour prendre le mal à la racine.

Réappropriation des préconstruits ethnologiques

Cet argumentaire selon lequel les pratiques nommées utani, senankuya, dendiragu, dakure, etc. constituent des modes traditionnels de résolution des conflits repose sur la réappropriation de la construction ethnologique de "tradition africaine » et sur sa réactualisation aux enjeux présents. En d'autres termes, les observateurs sont aujourd'hui les témoins du processus de construction d'une "nouvelle tradition " ou " néo-tradition " (Galvan 2003). Les pratiques de relations à plaisanteries, multiples et fluides, au cœur de la production et de la reproduction des frontières identitaires, sont en instance d'être institutionnalisées par les discours savants. La lecture univoque d'une histoire africaine mythifiée rend légitime cette institutionnalisation, cette réification des façons de faire locales, et affiche clairement ses objectifs idéologiques.

Faisant fi non seulement de la réalité historique des lieux d'émergence ponctuels de ces relations et des ressorts politiques qui les sous-tendent, mais aussi de leurs conditions opératoires en situation, à savoir l'existence de hiérarchies identitaires et politiques, nombre d'intellectuels et d'hommes politiques réduisent les relations à plaisanteries à des mécanismes d'évacuation des tensions psychologiques et sociales, à mi-chemin entre l'obédience à la théorie fonctionnaliste et la fidélité à la théorie symboliste (Hagberg 2002). En Afrique de l'Ouest, la référence aux travaux de Sory Camara (1969), dont l'attachement à ces deux perspectives ethnologiques est évident, est utilisée de manière quasi incontournable dans les argumentaires sur la fonction à faire jouer aux relations à plaisanteries. Pour Doulaye Konaté (2001: 3), «le sanankouya établit une relation pacificatrice qui joue le rôle d'exutoire de tensions qui autrement dégénèreraient en violences. Comme l'écrit fort justement Sory Camara, "il s'agit de désamorcer la guerre, de la jouer pour ne pas la faire" [...]. Le sanankouya agit comme une thérapeutique des situations qui ailleurs conduiraient à des conflits ouverts ». On ne peut manquer de constater la fidélité de l'auteur aux postulats de la catharsis individuelle et sociale. La puissance structurante de la "bibliothèque coloniale » sur la pensée africaine moderne est donc bien visible dans le cas des discours sur les relations à plaisanteries (Mudimbe $1988,1994)$. Une majorité d'intellectuels et d'hommes politiques semblent se contenter de s'approprier des discours pré-constitués, qui leur indiquent les limites de ce qui peut être énoncé (Mbembé 2000).

57 En outre, la mise en lumière de la solidarité entre groupes que les relations à plaisanteries contribueraient à construire et à maintenir évacue toute la problématique des relations dissymétriques de pouvoir qui fondent historiquement ces relations, et qui sont au cœur des pratiques actuelles. On a le sentiment que les auteurs tentent de minimiser la 
potentialité conflictuelle que les travaux d'autres chercheurs ont révélée dans les relations à plaisanteries en Afrique occidentale. Bakary Soumano (2002:3) a recours à l'oxymore de la «dérision cordiale» pour réconcilier la solidarité qu'il prône avec l'outrance réelle de certaines paroles. Il n'y a pas de grossièreté ni d'agressivité, mais des blagues et des farces sans conséquence: "Sont donc exclus du jeu, la médisance, la calomnie et autres comportements susceptibles de vicier l'atmosphère de convivialité du groupe. Au contraire, les taquineries qui cassent la glace d'un premier contact, les drôleries, les farces, le comique en général, toutes les actions humoristiques qui permettent de créer les conditions propices à l'accueil hospitalier de l'inconnu en instaurant une ambiance détendue, d'évacuer les conflits potentiels ou réels, bénins ou graves et de bâtir une paix sociale sereine et chaleureuse sont les bienvenus. » C'est donc par un aveuglement délibéré du fonctionnement réel des relations à plaisanteries, de leur jeu avec les identités locales et les hiérarchies de pouvoir qui les soutiennent, que les auteurs parviennent à réduire ces pratiques à un moteur dans la construction du lien social.

Il s'ensuit enfin que ces stratégies de construction d'États-nations fondés sur cette néotradition africaine reposent sur une idéologie de la cohabitation pacifique de groupes ethniques bien différenciés. La nation se réduirait à une agglomération de groupes qui entretiennent certes des relations cordiales et pacifiques, mais qui sont perçus et décrits comme foncièrement distincts les uns des autres. Il s'agit d'instituer du lien social dans une société multiculturelle, de préserver la paix à travers un discours de la différence ${ }^{37}$. Ce paradoxe a été mis en évidence par Etienne Smith (2004) dans son article sur le cousinage à plaisanteries au Sénégal. Essayant de dépasser les contradictions entre un discours universaliste qui prend appui sur des valeurs communes à tous les peuples et un discours particulariste qui renforce les différences à travers le jeu de la plaisanterie, lequel est avant tout une énonciation des stéréotypes qui caractérisent l'Autre et m'en distinguent, l'auteur soutient que ce n'est pas tant la transcendance des différences qui permet de tisser du lien social que l'acceptation d'un pluralisme pacifique, renégocié en permanence. Toutefois, on peut se demander si ce n'est pas justement dans les interstices de la renégociation que s'engouffrent les chantres de la différence, portés par la hantise des connexions entre groupes sociaux et de la fluidité des frontières identitaires. Faire de la fiction de l'ethnie le fondement moral de la solidarité politique, au lieu de concevoir des identités postcoloniales en devenir (Mbembé 2001) ne marque-t-il pas l'échec des nouveaux régimes narratifs qui tentaient de disqualifier les pré-construits coloniaux pour construire autrement les identités africaines?

L'Afrique comme espace démocratique

Si la tradition a longtemps été présentée comme ce qui participait à entretenir la fragilité des modes de gouvernance des sociétés africaines (la fidélité aux affiliations tribales, le respect des hiérarchies de pouvoir, la passivité face aux conditions de vie) ${ }^{38}$, elle est aujourd'hui réhabilitée par les acteurs intellectuels locaux. Ces derniers se présentent comme des porte-parole des valeurs et des pratiques qui seraient les seules réponses adéquates dans la lutte contre le désordre civil. Les tentatives de réhabilitation des traditions africaines dans l'espace politique reposent sur la permanence du constat fait par de nombreux intellectuels de l'échec de la « greffe » des techniques de règlement des conflits à l'occidentale en Afrique. L'instabilité permanente du pouvoir dans les sociétés africaines et les montées de violences sanguinaires de manière périodique amènent à envisager non seulement l'inefficacité des politiques inspirées du modèle démocratique 
européen, mais surtout leur inadéquation complète au modèle de développement africain. Autrement dit, ce constat de la particularité de l'espace africain est alimenté par une vision afrocentriste selon laquelle il y aurait une frontière étanche entre Afrique et Occident. Les sociétés africaines auraient développé des techniques n'existant nulle part ailleurs, dont feraient partie les relations à plaisanteries. Ces techniques seraient la preuve de l'indéniable unité du continent africain, unité basée sur la différenciation radicale avec l'autre (l'Occident). Sous l'expression de "savoirs locaux" (Hountondji 1994; Gyekye 1996) se cache l'idée d'une fermeture culturelle, sociale, politique et économique du continent africain au reste du monde. La revendication de l'authenticité africaine se présente en concurrence du modèle européen.

Au fur et à mesure de l'exposé des techniques de résolution des conflits, le lecteur voit se mettre en place une rhétorique de la réappropriation des valeurs démocratiques habituellement associées à l'espace européen. L'idée centrale sur laquelle repose cette rhétorique consiste à affirmer qu'aurait eu lieu une création endogène des principes démocratiques en Afrique. Ainsi, pour le griot Bakary Soumano (2002: 1), les relations à plaisanteries ne sont rien de moins qu'une forme de démocratie: "Les relations cathartiques sont un système de gestion de la diversité qui, en débordant les clans, les ethnies, les castes et les âges, propose une démocratie universelle et conviviale garantissant la dignité de l'autre en toute circonstance. " Les défenseurs des relations à plaisanteries participent donc de ce mouvement de réafricanisation qui consiste à trouver un ancrage local aux formes de gouvernance importées (Amselle 2001). Mais ils le poussent à l'extrême, puisqu'il faut comprendre dans les propos de Soumano que la démocratie et les Droits de l'Homme ne sont pas l'apanage des sociétés européennes. Il y aurait eu un surgissement en plusieurs points de la planète de la démocratie et des valeurs de justice et d'égalité. Qui sait si, dans cette conception, les valeurs qui soustendent les relations à plaisanteries ne seraient pas même antérieures à la naissance du modèle de la démocratie européenne en Grèce Antique ? ${ }^{39}$. La tendance à l'exaltation de l'Afrique se perçoit aisément à travers ce plaidoyer pour la démocratie africaine. Comme le défend Achille Mbembé (2000:27), elle témoigne de la difficulté à s'émanciper de la ratio occidentale. Il est vrai qu'en prônant l'idée que «l'Afrique a des choses à elle », tout aussi dignes de louanges que les valeurs et les pratiques occidentales, les intellectuels et les hommes politiques cités ici tentent en effet de rappeler « la participation de l'Afrique au travail de l'universel». Mais ils perpétuent la coupure entre Afrique et Occident, fidèles au mouvement de la négritude et au panafricanisme, et réactivent par-là même les discours coloniaux d'une différence infranchissable entre la « race » noire et le reste du monde.

Africanisme et dispositifs d'assujettissement

61 Le processus actuel de ré-imagination de la coutume dite relations à plaisanteries a pour objectif de l'investir d'un pouvoir nouveau, celui de perpétuer le lien social dans des pays qui vivent des périodes de déstabilisation récurrentes. Il convient toutefois de noter que ce vœu pieux consiste à confirmer l'idée que la fin justifierait les moyens. Déjà en 1960, I. Wallerstein soutenait la proposition que l'ethnie, cette fiction de la «raison ethnologique », pouvait être fonctionnelle du point de vue de l'intégration nationale. Cette idée se perpétue aujourd'hui à travers le concept de «bonne gouvernance » en politique, qui s'appuie sur la notion d'ethnie au lieu de prendre au sérieux son statut d'objet construit. La promotion des relations à plaisanteries dans le champ politique africain, soutenue par la science politique anglo-saxonne, relève d'une pensée analogue. 
Entretenir les fictions identitaires, les fonder sur des liens historiquement construits et considérés comme immuables, revendiquer leur caractère proprement africain et leur fonctionnalité dans la réduction des tensions ethniques, revient à imposer une conception unique de l'identité et de l'appartenance à la nation. Les récents articles des politologues américains Brett R. O'Bannon (2003) et Mark Davidheiser (2003) sur les relations à plaisanteries comme modes de prévention et de réduction des conflits témoignent de la difficulté de la science politique à prendre au sérieux la déconstruction anthropologique des identités : alors qu'elles indiquent clairement que les identités sont labiles, les analyses théoriques des auteurs et les réflexions sur des cas d'études de règlement de conflits renouent avec l'africanisme classique.

En outre, l'usage instrumental que proposent les défenseurs de la promotion des relations à plaisanteries dans le contexte moderne consiste, comme le dit Achille Mbembé (2000), à « détacher les pratiques de leur localité ", à les « ériger en technologies générales », selon une logique gouvernementale et non plus pratique. Il s'agit de sélectionner, dans la quotidienneté des pratiques variées et mouvantes, liées aux situations d'interactions, l'aspect le plus notoire et le plus utile afin de l'ériger en dispositif de discipline des populations. Certes, le caractère meurtrier des conflits en Afrique rend légitime des politiques de réduction de la violence. Mais la récupération politique des relations à plaisanteries soutenue par le déni de leur dimension pragmatique et agonistique, et leur manipulation à des fins de bonne gouvernance, interroge à nouveaux les rapports qui se tissent entre savoir (et refus de sa diffusion) et pouvoir politique.

64 Il a fallu s'attaquer à l'évidence et à la familiarité qui entourent le concept de « relations à plaisanteries » et en font un élément du sens commun ethnologique pour pouvoir opérer une relecture épistémologique des catégories de pensée et des paradigmes classiques de la discipline ethnologique. C'est en sortant du carcan des théories juridicofonctionnalistes et symbolistes, en s'éloignant des cohérences fonctionnelles, des systématisations formelles qui ensevelissent les rapports de pouvoir que les logiques qui fondent les pratiques regroupées sous l'étiquette de relations à plaisanteries par l'ethnologie classique ont pu être dévoilées. La naissance de la catégorie savante de relations à plaisanteries est venue supprimer toute référence aux rapports de force entre sociétés, pour lui substituer une vision romantique des groupes humains vivant en harmonie grâce à la passation de traités de paix respectés "à la lettre " ${ }^{40}$. Contre cette forme d'africanisme, le présent travail s'est donné pour objectif de traquer les enjeux politiques qui rendent compte de la production et de la reproduction des pratiques sociales en question. Il est apparu qu'en Tanzanie, à l'époque précoloniale, ces relations nées des frictions constantes entre unités sociopolitiques se caractérisaient par une dissymétrie dans la détention du pouvoir. Elles renvoyaient plus particulièrement aux formes d'association entre les groupes détenteurs du pouvoir militaro-politique et les groupes faiblement organisés. À l'époque coloniale, les relations à plaisanteries se sont perpétuées à travers les réaménagements des rapports de force entre les sociétés locales, et entre celles-ci et le pouvoir colonial. Enfin, aujourd'hui, les pratiques de relations à plaisanteries constituent des stratégies d'assise des positions de pouvoir par la réaffirmation des différences identitaires. Ces stratégies sont largement visibles dans les situations d'interaction.

Les discours politiques locaux qui se font entendre aujourd'hui se réapproprient la construction conceptuelle savante de relations à plaisanteries afin d'attester de 
l'existence de mécanismes indigènes anciens de règlement des conflits et de promotion de la solidarité interethnique. Il est évident qu'un tel discours est rendu possible par l'oubli des conditions de possibilité de la construction de ce concept, ainsi que des dissymétries de pouvoir sur lesquelles se sont échafaudés les modes d'interaction appelés relations à plaisanteries. En outre, il réactive la notion d'ethnie tant décriée par l'anthropologie, et ravive les flammes d'un afrocentrisme porté non seulement à exalter la différence, mais à la ré-inventer pour mieux l'imposer. On constate toutefois que les discussions sur le thème des relations à plaisanteries s'élargissent aujourd'hui à l'ensemble de la société civile. Différents types de discours apparaissent, proposant des visions alternatives au discours relativement homogène des intellectuels des années 1990-2000. Les débats futurs sur les relations à plaisanteries, qui témoigneront de la multiplicité des prises de parole dans des pays africains à la recherche d'un nouveau contrat social, viendront sans aucun doute relancer l'intérêt de l'anthropologie politique pour un objet jusqu'alors considéré comme classique et démodé.

* Cet article est tiré de la thèse de l'auteure (FouÉRÉ 2004b).

IFRA, Nairobi.

\section{BIBLIOGRAPHIE}

ABDY, D. C.

1924 « Notes on Utani and Other Bondei Customs », Man, 113-114 : 152-154, 164-166.

AMSELLE, J.-L.

1996a Vers un multiculturalisme français. L'empire de la coutume, Paris, Aubier.

1996b «L'étranger dans le monde mandingue et en Grèce Ancienne : quelques points de comparaison », Cahiers d'Études africaines, XXXVI (4), 144 : 755-761.

2001 Branchements. Anthropologie de l'universalité des cultures, Paris, Flammarion.

AMSElle, J.-L. \& M’BoKolo, E.

1985 Au cour de l'ethnie. Tribalisme et État en Afrique, Paris, La Découverte.

ASSEFA, $\mathrm{H}$.

1993 «Peace and Reconciliation as a Paradigm », Peace Initiative Series, 1, Nairobi : 5-16.

Austin, J.-L.

1962 How to do Things with Words, Oxford, Oxford University Press.

BADINI, A. 
1996 «Les relations de parenté à plaisanterie : éléments des mécanismes de régulation sociale et principe de résolution des conflits au Burkina Faso ", in R. OTAYEK, F. M. SAWADOGO \& J.-P. G UINGANE (dir.), Le Burkina entre révolution et démocratie (1983-1993), Paris, Karthala.

BARTH, F.

1969 Ethnic Groups and Boundaries. The Social Organisation of Culture Difference, Bergen-Oslo, Universitet Forlaget ; London, George Allen \& Unwin.

BATESON, G.

1977 Vers une écologie de l'esprit, Paris, Éditions du Seuil, 2 volumes.

BAUMAN, R.

1977 Verbal Art as Performance, Prospect Heights, IL, Waveland.

BAUMAN, R. \& SHERZER, J.

1974 Exploration in the Ethnography of Speaking, Cambridge, Cambridge University Press.

BAYART, J.-F.

1989 L'État en Afrique. La politique du ventre, Paris, Fayard.

BEIDELMAN, T. O.

1966 « Utani : Some Kaguru Notions of Death, Sexuality and Affinity », Southwestern Journal of Anthropology, 22 (4) : 354-380.

1997 The Cool Knife. Imagery of Gender, Sexuality and Moral Education in Kaguru Initiation Ritual, Washington D.C., Smithsonian Institute.

BOAS, F.

1898 «12th Report on the North-Western Tribes of Canada ", British Association for Advanced Science.

BOURDIEU, P.

1972 Esquisse d'une théorie de la pratique précédée de Trois études d'ethnologie kabyle, Paris, Éditions du Seuil.

1980 Le sens pratique, Paris, Éditions de Minuit.

1982 Ce que parler veut dire. Économie des échanges linguistiques, Paris, Fayard.

BOUVERESSE, J.

1973 Wittgenstein : la rime et la raison. Science, éthique et esthétique, Paris, Éditions de Minuit.

1976 Le mythe de l'intériorité, expérience, signification et langage privé chez Wittgenstein, Paris, Éditions de Minuit.

BRANDSTRÖM, P.

1986 "Who is a Sukuma and Who is a Nyamwezi. Ethnic Identity in West-Central Tanzania ", Working Papers in African Studies, 27, Uppsala University.

CAMARA, $\mathrm{S}$.

1969 Gens de paroles. Essai sur la condition et le rôle des griots dans la société malinké, thèse de doctorat, Université de Bordeaux, Faculté des lettres et Sciences Humaines.

CANUT, C. 
2002 « Pouvoirs, places et filiation. Les Senankuya au Mali », Cahiers de Praxéologie, 38 : 175-197.

Christensen, J. B.

1963 « Utani : Joking, Sexual Licence and Social Obligations among the Luguru », American Anthropologist, 65 (6) : 1314-1327.

von Clausewitz, C.

1832 «Vom Kriege », Hinterlassene Werke, Bd 1-2-3, Berlin (1955, De la guerre, Paris, Éditions de Minuit).

CLIFFORD, J.

1990 « Notes on (Field)Notes », in R. SANJEK (ed.), Fieldnotes. The Making of Anthropology, Ithaca \& London, Cornwell University Press.

DALE, G.

1896 « Account of the Principal Customs and Tribes of the Natives Inhabiting the Bondei Country ", Journal of the Royal Anthropological Institute : 181-239.

Dale, A. \& SMith, E. W.

1920 The Ila-speaking People of Northern Rhodesia, London, McMillan and Co.

DAVIDHEISER, M.

2003 «Joking Kinship and Conflict Management : An African Perspective on Dispute Mediation », paper presented for the American Anthropological Association Meetings in Chicago, April.

Delafosse, M.

1912 Haut-Sénégal et Niger, t. 2, Les civilisations, Paris, Larose.

DeleuZe, G.

1986 Foucault, Paris, Éditions de Minuit.

DieTERLEN, G.

1951 Essai sur la religion bambara, Paris, PUF.

DOKE, C. M.

1931 The Lambas of Northern Rhodesia. A Study of their Customs and Beliefs, London, Georg G. Harrap \& Co. Ltd.

DOUMBiA, P. E. N.

1936 « Étude du clan des forgerons ", Bulletin du Comité d'Études historiques et scientifiques de l'AOF, $19(2-3): 334-380$.

DOUMBiA, T.

2002 "Les relations à plaisanteries dans les sociétés mandingues ", Recherches africaines, Annales de la faculté des Lettres, Langues, Arts et Sciences humaines, Bamako, Université du Mali, janvier-juin.

DURANTI, A.

1997 Linguistic Anthropology, Cambridge, Cambridge University Press.

Duranti, A. \& GoODWIN, C. 
1992 Rethinking Context. Language as an Interactive Phenomenon, Cambridge, Cambridge University Press.

EVANS-PRITCHARD, E. E.

1971 La femme dans les sociétés primitives et autres essais d'anthropologie sociale, Paris, PUF (1965, The Position of Women in Primitive Society and Other Essays in Cultural Anthropology, London, Faber \& Faber).

FAY, C.

1995 " "Car nous ne faisons qu'un". Identités, équivalences, homologies au Maasina (Mali) ", Cahiers des Sciences humaines, ORSTOM, 31 (2) : 427-456.

FERGUSON, J. \& GUPTA, A. (eds.)

1997 Anthropological Locations : Boundaries and Grounds of a Field Science, University of California Press.

FOUCAULT, M.

1997 Il faut défendre la société, Paris, Gallimard-Éditions du Seuil.

FOUÉRÉ, M.-A.

2004a « La dimension politique de l'utani », in H. MAUPEU (dir.), L'Afrique orientale. Annuaire 2003, Paris, L'Harmattan.

$2004 \mathrm{~b}$ L'objet ethnologique « relations à plaisanteries » dans l'espace est-africain (Tanzanie). De la construction savante d'une coutume à la restitution des situations sociales de l'utani, Doctorat, Paris, École des hautes études en sciences sociales.

GALVAN, D.

2003 « Joking Kinship, Constructivism, and Ethnic Cooperation in Senegal », Paper for the presentation at the 2003 Annual Meeting of the African Studies Association, Boston, October.

GOFFMAN, E.

1987 Façons de parler, Paris, Éditions de Minuit.

1991 Les cadres de l'expérience, Paris, Éditions de Minuit.

Goody, J.

1979 La raison graphique. Domestication de la pensée sauvage, Paris, Éditions de Minuit.

GRAY, R. \& BIRMINGHAM, D.

1970 Pre-colonial African Trade. Essays on Trade in Central and Eastern Africa before 1900, London-New York, Oxford University Press.

GRIAULE, M.

1948 «L'alliance cathartique », Africa, $18: 242-258$.

GYEKYE, K.

1996 African Cultural Values: An Introduction, Accra, Sankofa Publishing Company.

HAFFENDEN, Capt.

1930 The Red Men of Nigeria, London.

HAGBERG, S. 
2002 "The Politics of Joking Relationships in Burkina Faso », Paper presented at the workshop on «Friendship, Descent and Alliance », 16-18 December 2002, Max Planck Institute for Social Anthropology, Germany.

HOBSBAWM, E. \& RANGER, T.

1983 The Invention of Tradition, Cambridge, Cambridge University Press.

HoUnTONDJI, P.

1994 Les savoirs endogènes. Pistes pour une recherche, Dakar, CODESRIA ; Paris, Karthala.

Humblot, P.

1918 « Du nom chez les Malinkés des vallées du Niandou et du Milo », Bulletin du Comité d'Études historiques et scientifiques de l'AOF : 519-540.

ILIFFE, J.

1969 Tanganyika under German Rule, London, Cambridge University Press.

1979 A Modern History of Tanganyika, Cambridge, Cambridge University Press.

JUNOD, H. A.

1962 [1913] The Life of a South-African Tribe, New Hyde Park, N.Y., University Books.

KJEKSHUS, $\mathrm{H}$.

1977 Ecology Control and Economic Development in East African History. The Case of Tanganyika, 1850-1950, London, Heinemann.

KONATÉ, D.

2001 « Les fondements endogènes d'une culture de la paix au Mali : les mécanismes traditionnels de prévention et de résolution des conflits ", in Les fondements endogènes d'une culture de la paix en Afrique : Mécanismes traditionnels de prévention et de résolution des conflits, Paris, UNESCO, http:// www.unesco.org/cpp/publications/mécanismes/edkonate.htm.

KOPONEN, J.

1988 People and Production in Late Precolonial Tanzania. History and Structures, Helinski, Finish Society for Development Studies.

KoPYTOFF, I.

1987 The African Frontier. The Reproduction of African Society, Bloomington, Indiana University Press.

LABOURET, $\mathrm{H}$.

1929 «La parenté à plaisanteries en Afrique Occidentale », Africa, 2 : 244-254.

1934 Les Manding et leur langue, Paris, Larose.

LAUNAY, R.

1977 « Joking Slavery in Africa », Africa, 47 (4) : 411-422.

LOWIE, R. H.

1912 "Social Life of the Crow Indians ", Anthropological Papers of the American Museum of Natural History, 9, part 2.

LUCAS, S. 
1974 Utani Relationships in Tanganyika, 7 vol., Université de Dar es-Salaam.

MALINOWSKI, B.

1922 Argonauts of the Western Pacific. An Account of Native Entreprise and Adventure in the Archipelagoes of Melanesian New Guinea, London, Routledge \& Sons Ltd.

MAUSS, M.

1924 «Essai sur le don. Forme et raison de l'échange dans les sociétés archaïques », in M. MAuss, Sociologie et anthropologie, Paris, PUF.

1928 « Parenté à plaisanteries », in M. MAuss, Essais de Sociologie, Paris, Éditions de Minuit.

MBEmBÉ, A.

2000 «À propos des écritures africaines de soi », Politique Africaine, 77 : 16-43.

2001 On the Postcolony, Berkeley-Los Angeles-London, University of California Press.

MCVICAR, Rev.

1935 « Sibs, Privileged Familiarity and Cross-Cousin Marriage among the Waluguru », Primitive Man, 8 : 57-67.

MEEK, C. K.

1925 The Northern Tribes of Nigeria : An Ethnographical Account of the Northern Provinces of Nigeria together with a Report on the 1924 Decennial Census, London, Cambridge University Press.

MELLAND, F. H.

1967 [1923] In Witch-Bound Africa. An Account of Primitive Kaonde Tribe and Their Beliefs, London, Franck Cass \& Co. Ltd.

MKANGI, K.

1997 «Indigenous Social Mechanism of Conflict Resolution in Kenya. A Contextualized Paradigm for Examining Conflict in Kenya », http://www.payson. tulano.edu/conflicts/Cs\%20St/ MKACON2.html.

MOREAU, R. E.

1941 « The Joking Relationships in Tanganyika », Tanganyika Notes and Records, 12 :1-9.

1944 «Joking Relationships in Tanganyika », Africa, 14 (7) : 386-400.

MUdimBe, V. Y.

1988 The Invention of Africa, Bloomington, Indiana University Press.

1994 The Idea of Africa, Bloomington, Indiana University Press.

O’BANNON, B. R.

2003 « Do not Speak to Me of Cousinage : Neoliberalism, Changing Identities and Conflict Management in Senegal », Paper presented at the African Association Annual Meeting, Boston, October.

PARKIN, D.

1980 " The Creativity of Abuse », Man, $15: 45-64$.

PAulme, D. 
1939 « Parenté à plaisanteries et alliances par le sang en Afrique occidentale », Africa, 12 :

433-444.

PEDLER, F. J.

1940 «Joking Relationships in East Africa », Africa, 13 : 170-173.

RADCLIFFE-BROWN, A. R.

1940 « On Joking Relationships », Africa, $13: 195-210$

1949 « A Further Note on Joking Relationships », Africa, 19 : 133-140.

1952 Structures and Functions in Primitive Society, Glencoe, Free Press.

REICHAR, P.

1890 « Die Wanjamuesi », Deutsche Kolonialzeitung : 228-230, 239-241, 262-265, 276-278.

RICHARDS, A. I.

1937 "Reciprocal Clan Relationships among the Bemba of Northern East Rhodesia ", Man, XXXVII (222) : 188-193.

ROBERTS, A.

1970 « Nyamwezi Trade », in R. Gray \& D. Birmingham (eds.), Pre-colonial African Trade. Essays on Trade in Central and Eastern Africa before 1900, London-New York, Oxford University Press.

SAKHO, A.

2002 Propos rapportés dans l'article de Baba Thiam, « Senégal : Diversité et tolérance, facteurs de paix » (http://panos.sn/panosinfos/diverssn_paix3.htm).

SANJEK, R.

1990 Fieldnotes. The Making of Anthropology, Ithaca \& London, Cornwell University Press.

SCRIVENOR, T. V.

1937 "Some Notes on Utani, or the Vituperative Alliances Existing Between Clans in the Masasi District », Tanganyika Notes and Records, 4 : 72-74.

SEARLE, J. R.

1969 Speech Acts, Cambridge, Cambridge University Press.

SISSAO, A. J.

2002 Alliances et parentés au Burkina Faso : mécanismes de fonctionnement et avenir, Ouagadougou, Sankofa et Gurli Éditions.

SMITH, E.

2004 « Le cousinage de plaisanterie en Afrique de l'Ouest, entre particularismes et universalismes ", Raisons politiques, $13: 157-169$.

SOUMANO, B

2002 L'éthique des relations cathartiques, document dactylographié.

STEFANISZYN, père B.

1964 Social and Ritual Life of the Ambo in Northern Rhodesia, Oxford, Oxford University Press.

TAMARI, T. 
1997 Les castes de l'Afrique occidentale. Artisans et musiciens endogames, Nanterre, Société d'Ethnologie.

TANOU, $\mathrm{S}$.

2003 « Burkina Faso Joking Relationship », ANB-BIA Supplement, 453, http://www.peacelink.it/ anb-bia/nr453/e02.html.

WALLERSTEIN, I.

1960 « Ethnicity and National Integration in West Africa », Cahiers d'Études africaines, 3 : 129-139.

WEGRU, J. Y.

1999 « The Dagaaba-Frafra Joking Relationship », Folklore, 14, décembre, www.folklore.ee/ folklore/vol14/dagaaba.htm.

WHITE, C. M. N.

1955 «Joking Relationships in Central Africa », African Studies, 14 (3) : 97-112.

WILSON-FALL, W.

2000 "Conflict Prevention and Resolution among the Fulbe », in I. W. ZARTMAN (ed.), Traditional Cures for Modern Conflicts : African Conflict « Medicine », Boulder, CO ; London, Lynne Rienner.

WiTTGENSTEIN, L.

1985 Investigations philosophiques, Paris, Gallimard.

ZARTMAN, I. W.

1985 Ripe for Resolution. Conflict and Intervention in Africa, New York-Oxford, Oxford University Press.

1997 Governance as Conflict Management : Politics and Violence in West Africa, Washington D.C., Brookings Institute Press.

2000 Traditional Cures for Modern Conflicts : African Conflict « Medicine », Boulder, CO ; London, Lynne Rienner.

\section{NOTES}

1. L'orthographe de ce terme varie selon les auteurs : séninkougna, sanankuya, sanaku ya, sanakunya, etc.

2. Cette expression a été forgée par l'anthropologue américain R. H. LoWIE (1912) dans ses travaux des années 1910 sur les Indiens Crow d'Amérique du Nord.

3. Les relations à plaisanteries intrafamiliales sont couramment appelées " parenté à plaisanteries » (MAUSS 1928).

4. Les relations à plaisanteries interclaniques et interethniques sont parfois appelées « cousinage à plaisanteries » (CAMARA 1969). Ces deux types de relations sont distinguées dans la présentation qui suit, car elle récapitule les perspectives de l'ethnologie classique, fondées sur la distinction entre les modes d'organisation sociopolitiques (lignages, clans et ethnie). En s'inspirant des réflexions de déconstruction des notions d'« ethnie » et de « clan », une telle distinction sera ici critiquée.

5. En swahili, « partenaire à plaisanteries » se dit mtani (sg.), watani (pl.).

6. Aussi parus dans son ouvrage Stuctures and Functions in Primitive Society (1952). 
7. Constatons à ce propos qu'un tel argumentaire repose sur le présupposé d'une nature humaine belliqueuse par instinct.

8. «Utani exists on an intertribal as well as intratribal level, and serves much the same function of dissipating hostility and maintaining harmony between groups » (CHRISTENSEN 1963 : 1314-1327).

9. EVANS-PRITCHARD (1971: 68) note avec ironie la répulsion qu'éprouve le Blanc pour l'obscène chez les autres : «Cependant, la plupart des Européens, bien qu'incapables de découvrir les obscénités de leur propre société, les perçoivent parmi les indigènes parce qu'elles leur sont étrangères, et les condamnent sans appel. Il semble que le sens moral du Blanc, offensé par ces obscénités, rende compte de telles condamnations [...]. On les décrit comme "au-delà de la bestialité" ou "trop horribles" pour être répétées. "

10. Le mode de fonctionnement du royaume mis en place autour des années 1870 par le chef Nyamwezi Mirambo illustre les rapports de pouvoir dissymétriques entre unités sociales de Tanzanie. La puissance de ce chef reposait sur la fidélité de ses guerriers, nommés ruga ruga, qui parcouraient les territoires aux alentours de la ville qu'il avait fondée, dans le but de piller les ressources des villages d'agriculteurs ou le bétail des populations d'éleveurs. Les populations les plus proches du cœur du royaume, lorsqu'elles ne fuyaient pas leurs terres, acceptaient la domination de ce royaume en payant un tribut régulier.

11. «Plutôt que de partir du sujet (ou même des sujets) et de ces éléments qui seraient préalables à la relation et qu'on pourrait localiser, il s'agirait de partir de la relation même de pouvoir, de la relation de domination dans ce qu'elle a de factuel, d'effectif, et de voir comment c'est cette relation elle-même qui détermine les éléments sur lesquels elle porte. Ne pas donc demander aux sujets comment, pourquoi, au nom de quels droits ils peuvent accepter de se laisser assujettir, mais montrer comment ce sont les relations d'assujettissement effectives qui fabriquent des sujets » (FoucAult 1997 : 38).

12. « Utani is a system of ritual interaction closely linked to cultural and socio-economic differentiation between the groups and the individuals who practice it. [It] provides an ideological framework which justifies differential access to resources while affirming social equality of the participants » (LUCAS $1974: 12$ ).

13. Certes, Foucault s'intéresse aux sociétés européennes, de sorte qu'il faut prendre garde de ne pas transposer ses réflexions sans tenir compte des différences de situations selon les lieux et les époques considérés. Toutefois, la portée des argumentations qu'il propose me semble si large que leur pertinence ne fait pas de doute dans l'analyse du fonctionnement des rapports de force au Tanganyika à l'époque précoloniale. DELEUZE (1986 : 43) a souligné combien la transposition des réflexions de Foucault aux sociétés « primitives » s'avère pertinente : « Car, loin d'être sans politique et sans histoire, [les sociétés dites primitives] ont un réseau d'alliances qui ne se laissent pas déduire d'une structure de parenté, ni réduire à des relations d'échanges entre groupes de filiation. Les alliances passent par des petits groupes locaux, constituent des rapports de force (dons et contre-dons) et conduisent le pouvoir. »

14. Je renvoie aux réflexions critiques de James FERGUSON et d'Akhil GUPTA (1997) sur l'archétype malinowskien du «terrain » comme observation directe de l'Autre dans son contexte de vie « naturel », c'est-à-dire hors de tout contact avec des cultures différentes, et en particulier avec le monde urbain.

15. J'insiste sur le fait que les interactions dont j'ai été le témoin sont, pour les interlocuteurs, des situations d'utani. En effet, dans l'optique suivie, le travail du chercheur n'est pas de supputer ce qui peut être de l'utani et ce qui n'en est pas, mais de partir des 
assignations de ce signifiant à certaines situations par les interlocuteurs, pour saisir les logiques de « cadrage » (GOFFMAN 1991) des situations et les logiques d'émergence des paroles et des pratiques ainsi cadrées.

16. J'ai pris note de ces situations d'interaction non pas directement au moment de leur émergence, mais peu de temps après, généralement le soir. Mes notes de terrain sont donc le résultat d'une « inscription », pour reprendre la terminologie de James CLIFFORD (1990), c'est-à-dire des productions écrites qui ont pour objectif de servir d'« aidemémoire » (SANJEK 1990) au moment de se remémorer un fait observé et son contexte d'observation, pour les rédiger plus amplement. Les événements ici décrits ne sont pas directement les notes de terrain, peu lisibles pour celui qui ne les a pas écrites. Il s'agit d'une mise en forme de ces notes (ce que Clifford nomme l'étape de " description ») destinée à présenter au lecteur de manière cohérente les faits observés. Elle n'est possible que par la conjugaison des notes de terrain et des " notes de mémoire ", ou " headnotes " (SANJEK 1990), ces résidus de l'expérience de terrain enregistrés dans la mémoire, que la lecture de notes écrites, aussi ténues soient-elles, permet de stimuler.

17. «Mimi Mnyamwezi. Tuongee kinyamwezi» (« le kinyamwezi est la langue des populations dites wanyamwezi »).

18. «Wanyamwezi hawaongei. »

19. «Wewe! Naona unapenda utani !"

20. «Anapenda kumtania tu.»

21. «Huyu si mtani wangu, wanafanya kazi shambani kwetu. Sisi bossi wao.»

22. Parmi une multitude de travaux, voir les ouvrages fondateurs: WITTGENSTEIN (1985), A ustin (1962), SEARLE (1969), mais aussi BouRdieu $(1980,1982)$, BAUMAN (1977), BAUMAN \& S HERZER (1974), DURANTI (1997), DURANTI \& GOODWIN (1992).

23. L'expression « fonction dénotative » renvoie à la fonction de description ou de constatation de la langue.

24. Par « jeux de langage », Wittgenstein entend qu'il est possible de déterminer diverses catégories d'énoncés (renvoyant à ce que d'autres appellent les « fonctions » de la langue) par l'étude des règles qui spécifient leurs propriétés et l'usage qu'on peut en faire (de la même manière que le jeu d'échec se définit par des règles sur les propriétés des pièces et les modalités de leurs déplacements). Sur ce point, voir les travaux de BOUVERESSE (1973, 1976).

25. Par fonction performative, on entend que certains types d'énoncés ont la particularité de faire advenir ce qu'ils énoncent.

26. "Nimekudanganyia, bagia nimezinunua hapa »/« mimi mtani wako.»

27. «Mbona unanisema, wewe mtani wangu?».

28. "Ndo tabia yao kwetu.»

29. Sur la construction des statuts et l'institution des castes, voir par exemple TAMARI (1997).

30. Dans un article qui aborde la question des relations à plaisanteries, David PARKIN (1980) se situe dans cette perspective analytique des pratiques sociales. Il souligne la dimension stratégique des échanges langagiers, la « créativité » sémantique qui les caractérise. Il convient selon lui de ne pas analyser en termes d'échanges ritualisés, de codification, donc de tradition et de normes intangibles, les formes de salutations, d'adresse, d'adieu, et de relations à plaisanteries. Il défend l'idée que même s'il existe un ensemble d'attentes sociales normatives, les individus les adaptent à la situation donnée 
afin de retourner celle-ci à leur avantage («joking relationship [...] hints at flexibility in social rules and their possible re-negociation $»$.

31. Le terme de « métacommunication », forgé par BATESON (1977), désigne une communication sur la communication, c'est-à-dire un ensemble d'informations qui renseignent sur la façon dont le message doit être interprété.

32. " siyo utani, sipendi utani, utani na biashara ndio tofauti."

33. Voir aussi Sarah TANOU $(2003: 4)$ qui défend le déploiement des relations à plaisanteries au Burkina Faso : «A joking relationship is therefore a precious fall-back in African traditional society. It should endeavour to preserve it carefully, for it is an instrument for the resolution of conflict. »

34. Voir par exemple le site gouvernemental :http://www.culture.gov.bf/M.C.A.T/ tourisme/tourisme_coutumes.htm.

35. Voir les débats sénégalais (http://panos.sn/panosinfos.htm) et l'article de Sten $\mathrm{H}$ AGBERG (2002) qui évoque la médiatisation des relations à plaisanteries au Burkina Faso.

36. «Utani: In this mechanism, a number of communities made permanent peace treaties among themselves. They were bound to offer support and protection to their respective members who happen to be within their respective territory. Such relations existed between contiguous communities, such as the Wakamba and Wataita of Kenya. But in some cases, even between some which where very far apart, such as the Wazaramo and Wanyamwezi of Tanzania » (MKANGI $1997: 3$ ).

37. Les travaux de Doulaye KonATÉ (2001 : 3) prônent l'intégration des connaissances sur les relations à plaisanteries dans les programmes scolaires. Dans son paragraphe intitulé « Des méthodes et techniques d'introduction des éléments de la culture traditionnelle dans les curricula ", il défend l'idée que la sanankounya peut facilement être enseignée « si une telle relation existe entre des ethnies ou des patronymes vivant dans le village ou la commune ». Dans un article sur les relations à plaisanteries au Ghana, J. Y. WEGRU (1999 : 5) propose d'entretenir les liens noués entre les populations gurune et les populations dagaaba par la promotion des mariages mixtes.

38. Voir les critiques de J.-F. BAYART (1989) adressées aux théories développementalistes. 39. Comme le suggère Katama MKANGI (1997 : 1), les valeurs de solidarité en Afrique étaient présentes bien avant que les organismes internationaux ne viennent préserver le lien social par une gestion financière : « [The principle of reciprocity] was a form which transformed individual obligations into social welfare security schemes (today's pension schemes and the so-called safety-nets which are now being "discovered" by the World Bank and IMF.»

40. C'est par ironie que cette expression a été ici choisie, puisque la majorité des sociétés dites exotiques n'avaient pas de système d'écriture. Pour de nombreux auteurs, cela vient confirmer la variabilité bien plus grande des formes d'organisation sociales (Goody 1979), et l'inadéquation de la notion même de « contrat " pour penser les modes d'agencement entre sociétés (HOBSBAWM \& RANGER 1983 ; AMSELLE \& M’BOKOLO 1985). 


\section{RÉSUMÉS}

L'adoption d'une approche politique, c'est-à-dire axée sur la recherche des rapports de force qui se trouvent au cœur des pratiques sociales, a permis de déconstruire la catégorie ethnologique de « relations à plaisanteries ». Cette catégorie a longtemps été appréhendée selon une perspective fonctionnaliste qui privilégie la cohérence du système sur les conflits de pouvoir qui s'exercent dans un espace social. Au contraire, la présente analyse de la production et de la reproduction des pratiques de « relations à plaisanteries » dévoile les logiques politiques et identitaires qui les sous-tendent. Elle souligne les enjeux actuels de l'usage de cette catégorie en Afrique.

The Metamorphoses of "Joking Relationships": A New Political Issue in Building Nation-States. - The present article is a contribution to the understanding of the notion of "joking relationships" from a political point of view. It aims at questioning the notion itself and at unveiling the relations of power that stand at the core of social practices. Instead of dealing with the object of "joking relationships" as if it were practices that could help to maintain a coherent social system, the article is concerned with the production and reproduction of such practices in relation to the workings of power in everyday life and the affirmation of ethnic identity. It emphasizes presentday issues that are linked to the manipulation of such a category in African countries.

\section{INDEX}

Keywords : history, joking relationships

Mots-clés : historicité, analyses langagières, déconstruction, postmodernité, rapports de pouvoir, réappropriation conceptuelle, relations à plaisanteries, speech analysis, deconstruction, postmodernity, workings of power, conceptual reappropriation 